\title{
Identification and Profiling of microRNAs and Their Target Genes in Tibetan Hulless Barley in Response to Barley Leaf Stripe (BLS) Infection
}

\section{Xiaohua Yao}

Qinghai University

\section{Yue Wang}

Qinghai University

Youhua Yao

Qinghai University

Yixiong Bai

Qinghai University

\section{Kunlun Wu}

Qinghai University

Youming Qiao ( $\nabla$ qhyoumingqiao@126.com )

Qinghai University

\section{Research}

Keywords: Tibetan hulless barley barley, barley leaf stripe, miRNA, target genes, small RNA sequencing

Posted Date: September 1st, 2020

DOI: https://doi.org/10.21203/rs.3.rs-66842/v1

License: (c) (i) This work is licensed under a Creative Commons Attribution 4.0 International License. Read Full License 


\section{Abstract \\ Background}

Tibetan hulless barley is widely grown on the Qinghai-Tibet Plateau, where it has served as a staple food for Tibetan people since the 5th century CE. Barley leaf stripe (BLS) is one of the most severe fungal diseases affecting the yield and quality of Tibetan hulless barley.

\section{Results}

Here, we compared the miRNA profiles before and after BLS in two Tibetan hulless barley genotypes: Z1141, a BLS-sensitive wild variety, and Kunlun14, a BLS-tolerant hybrid variety. A total of 36 conserved and 56 novel miRNAs were identified. Of these, 10 conserved and 10 novel miRNAs exhibited significantly changed expression between the normal and infected leaves of Kunlun14, respectively, while 3 conserved and 5 novel miRNAs exhibited significantly changed expression between the normal and infected leaves of Z1141, respectively. A total of 24 miRNAs were found in Z1141 and Kunlun14, and a further 546 putative target genes were predicted. Transcriptome sequencing analysis showed that among the 546 candidate genes, 131 had significant differences in expression between the normal and infected leaves of Kunlun14 and Z1141. Gene ontology, pathway, and Blast analyses indicated 10 candidate target genes that were involved in the barley disease resistance. These 10 candidate target genes may be regulated by 7 miRNAs. According to quantitative real-time PCR results, the 10 targets were negatively correlated with their corresponding miRNAs after infection with BLS.

\section{Conclusions}

The miRNAs and their target genes expressed in Tibetan hulless barley were identified and found to be associated with BLS resistance. Thus, these miRNAs and their target genes may be exploited via breeding programs or genetic engineering to improve BLS resistance in Tibetan hulless barley.

\section{Background}

Tibetan hulless barley (Hordeum vulgare L. var. nudum Hook. f.) is a self-reproducing annual species that produces naked grains. This plant is widely cultivated on the Qinghai-Tibet Plateau and has served as a staple food for the Tibetan people since the 5th century CE [1, 2]. Grains of Tibetan hulless barley not only contain abundant nutritional components and physiologically-active compounds, but also are rich in edible pigments necessary the food manufacturing industry $[3,4]$.

Barley leaf stripe (BLS) is a serious fungal disease affecting the yield and quality of Tibetan hulless barley in the Qinghai-Tibet plateau [5]. BLS is generally carried by grains and infects the leaves, sheaths, and spikes of host plants[6]. BLS is common in the main cultivation areas of Tibetan hulless barley in 
China, including Tibet, Qinghai, Gansu, Sichuan, and Yunnan [7]. Plant resistance of BLS is considered a complex trait, involving many disease resistance genes $[8,9,10]$. Quantitative trait locus (QTL) analysis is an efficient way to analyze some key traits when breeding for barley disease resistance [11, 12]. For example, applying QTL analysis to progeny crossed by BLS-resistant and -susceptible varieties identified two disease resistant genes, Rdg1a and Rdg2a, which are located on the $2 \mathrm{HL}$ and $7 \mathrm{HS}$ chromosomes, respectively $[8,9,13,14]$. Overexpression of these two genes markedly enhanced the resistance of barley to BLS $[15,16,17]$. However, only a few genes have been discovered via QTL analysis. This suggests that we may have reached a bottleneck in the study of BLS through traditional gene mapping analysis, and thus new methods and perspectives are needed to explore novel plant genes directly or indirectly related to $\mathrm{BLS}$ resistance and resistance mechanisms.

MicroRNA (miRNA) is a type of non-coding small RNA consisting of 21-24 nt that plays various critical roles in the growth and development of plants, including how plants respond to abiotic and biotic stressors, such as pests and diseases $[18,19,20]$. High-throughput sequencing of microRNA has become a fast, efficient, informative, and cost-effective strategy for gene discovery $[21,22,23]$. Some miRNAs and their target genes contribute critically to regulating plant disease. For example, miR393 responds to bacterial disease by negatively regulating the auxin receptor genes TIR1, AFB2, and AFB4 [24]. Some artificial miRNAs (amiRNAs) in barley participate in regulating yellow dwarf disease under low temperature stress conditions [25], while in tomato plants, miR403 can regulate leaf-shortening disease by targeting the AGO2 gene [26]. Researchers analyzed miRNA the changes in cotton roots infected by verticillium wilt and found that the expression levels of at least 65 miRNAs changed, most of which were non-conservative miRNAs [27]. It was discovered that viral-inducible OsAG018 sequesters miR168 to ease the repression of rice $O s A G O 1$ by miR168 to enable antiviral defense in infected rice [28] .

With the development of high-throughput sequencing, increasing numbers of plant miRNAs are being discovered. We are currently aware of 47,618 mature miRNAs covering 271 species, of which 10,414 mature miRNAs from 82 plant species have been identified in the miRNA database (http://www.mirbase.org/V21.0). Among the latter, 812 miRNAs originate from Arabidopsis, 325 from maize, 738 from rice, 241 from sorghum, and 126 from wheat. By contrast, only 71 miRNAs have been found in barley, and not a single miRNA has yet been reported from Tibetan hulless barley. Here, to fill this knowledge gap and explore disease-resistant miRNAs, high-throughput sequencing was used to construct a miRNA database of disease resistance and susceptible Tibetan hulless barley varieties. From our results, eight miRNAs and 10 candidate target genes related to BLS resistance were found and their expression patterns were analyzed in detail. Our study results not only provide a data foundation for the functional verification of BLS resistance genes, but also provide information for the future selection and breeding of new disease-resistant Tibetan hulless barley varieties.

\section{Materials And Methods}

\section{Plant materials and BLS treatment.}


Wild Tibetan hulless barley accession 'Z1141' and the hybrid variety 'Kunlun14' were used to investigate BLS resistance. The proportion of field area (per square meter) of Kunlun 14 and Z1141 was calculated from 2015 to 2017 . Kunlun14 is a cultivar that can tolerate BLS, while Z1141 is susceptible to BLS (Fig. 1). In this study, we used ZS, ZN, KL14S, and KL14N, as experimental materials. First, the infected leaves of Z1141 were collected to construct the infection system of BLS in the laboratory. To complete this process, five pieces $(5 \mathrm{~mm} \times 5 \mathrm{~mm})$ of one typical infected leaf from Z1141 were isolated, rinsed with $75 \%$ alcohol for $30 \mathrm{~s}$ and $2 \%$ sodium hypochlorite for $30 \mathrm{~s}$, and then washed with distilled water three times. Finally, they were cultivated in a sealed Petri dish filled with potato dextrose agar (PDA) culture medium (200 g of dissolved peeled potato, $17 \mathrm{~g}$ of glucose, $16 \mathrm{~g}$ of agar to $1000 \mathrm{~mL}$ with distilled water) after drying off. The dish was placed in a biochemical incubator at $22-25^{\circ} \mathrm{C}$ for $3 \mathrm{~d}$. Non-contaminated mycelia were transferred to a new PDA culture dish four times until the colony was purified. First, three ensuing fungal cakes were removed with a perforator and cultivated in another new PDA culture medium, which was then placed in a biochemical incubator at $25^{\circ} \mathrm{C}$ for $5-7 \mathrm{~d}$ until the whole Petri dish was filled with the BLS colony. Second, 300 grains of each Tibetan hulless barley were disinfected with $75 \%$ alcohol for $1 \mathrm{~min}$, and then 150 grains were equally placed into three Petri dishes covered with the BLS colony, and another 150 grains were equally placed in three Petri dishes covered with the PDA culture medium without BLS colony. The above six dishes were then placed into a low-temperature incubator for two weeks at $6{ }^{\circ} \mathrm{C}$. Finally, 300 grains of each variety were respectively planted in six flowerpots with vegetative soil and cultured in an artificial climate incubator (daytime: $14 \mathrm{~h}$, at $20^{\circ} \mathrm{C}$; nighttime: $10 \mathrm{~h}$, at $10^{\circ} \mathrm{C}$ ) for approximately $10 \mathrm{~d}$. The second infected or normal leaf from the bottom of the seedling was removed, and leaves with at least three biological repeats were refrigerated at $-80^{\circ} \mathrm{C}$ after freezing in liquid nitrogen.

\section{Small RNA library construction and sequencing.}

The total RNA of each leaf was extracted using TRIzol reagent (Invitrogen, CA, USA) according to the manufacturer's instructions, and the RNA purity was assessed using a NanoPhotometer ${ }^{\circledR}$ spectrophotometer (IMPLEN, CA, USA). The RNA concentrations were then measured using the Qubit $\circledast$ RNA Assay Kit in a Qubit ${ }^{\circledR}$ 2.0 Fluorometer (Life Technologies, MD, USA). The RNA integrity of each sample was assessed using the RNA Nano 6000 Assay Kit of the Agilent Bioanalyzer 2100 system (Agilent Technologies, CA, USA). The RNA integrity was further checked through a $1 \%$ agarose gel analysis. All RNA samples were then sent to Novogene (Beijing, China) to construct libraries for small RNA sequencing, where single-end reads (SE50) were sequenced on an Illumina HiSeq 2500 platform (Illumina Inc., CA, USA).

A total amount of $3 \mu \mathrm{g}$ total RNA per sample was used as the input material for the small RNA library. Sequencing libraries were generated using the NEBNext®Multiplex Small RNA Library Prep Set for Illumina ${ }^{\circledR}$ (NEB, USA), following the manufacturer's recommendations, with index codes added to label each sample with its corresponding sequences. PCR amplifications were performed using the Long Amp Taq $2 X$ Master Mix, SR Primer for Illumina, and index $(X)$ primer. The ensuing PCR products were then purified by $8 \%$ polyacrylamide gel analysis. DNA fragments corresponding to $140-160 \mathrm{bp}-$ the length of 
small non-coding RNA plus the $3^{\prime}$ and 5 ' adaptors-were recovered and dissolved in $8 \mu \mathrm{L}$ elution buffer. Lastly, library quality was assessed on the Agilent Bioanalyzer 2100 system using DNA High Sensitivity Chips. The clustering of index-coded samples was performed on a cBot Cluster Generation System that used the TruSeq SR Cluster Kit v3-cBot-HS (Illumina), following the manufacturer's instructions. After completing the cluster generation, the library preparations were sequenced on an Illumina HiSeq 2500/2000 platform, and 50-bp single-end reads were generated.

\section{Prediction of miRNA.}

The small RNA tags were mapped to reference sequences by Bowtie [29], with mismatching not allowed, in order to analyze their expression and distribution vis-à-vis the barley reference genome (ftp://ftp.ensemblgenomes.org/pub/plants/release-

36/fasta/hordeum_vulgare/cds/Hordeum_vulgare.Hv_IBSC_PGSB_v2.cds.all.fa.gz) [30]. These reference-mapped small RNA tags were then used to search for known miRNA. The miRBase 22 served as the reference, with modified mirdeep2 [31] and srna-tools-cli software [32] used to obtain the potential miRNA and to draw the secondary structures. Custom scripts were used to obtain the miRNA counts as well as the base bias on the first position of an identified miRNA with a certain length and on each position of all identified miRNA, respectively.

Hairpin structural characteristics of the miRNA precursor may be used to predict novel miRNA. The available software, miREvo [33] and mirdeep2 [31], were integrated to predict novel miRNAs by exploring their secondary structure, dicer cleavage site, and minimum free energy of the small RNA tags nonannotated in the prior steps above. The occurrence of miRNA families identified from the samples in other species was then explored. In this analysis pipeline, for a known miRNA, miFam.dat 22 (http://www.mirbase.org/ftp.shtml) was used to search for families; likewise, a novel miRNA precursor was submitted to Rfam 32.0 (http://pfam.xfam.org/) to identify Rfam families.

\section{Identification of differentially expressed miRNAs.}

The miRNA expression levels were quantified as transcripts per million (TPM) using these criteria [34, 35]: normalization formula: normalized expression $=$ mapped readcount $/$ total reads $* 1000000$. Differential expression analysis of the four treatment groups was performed in the R software environment using the DESeq package (v1.8.3), for which the $P$-values were adjusted using the Benjamini \& Hochberg method [36]. A corrected $P$-value of 0.05 was set, by default, as the threshold for a statistically significant level of differential gene expression.

\section{Prediction of miRNA target genes, GO and KEGG pathway analysis.}

Predicting the target gene of a given miRNA was carried out using psRNATarget for plants (http://plantgrn.noble.org/psRNATarget/) [37]. GO enrichment was conducted to analyze the likely functions of genes targeted by their miRNAs using the GO enrichment tool in PlantRegMap (http://plantregmap.cbi.pku.edu.cn/) [38]. Pathway analysis was performed using the Kyoto 
Encyclopedia of Genes and Genomes (KEGG) database (https://www.genome.jp/kegg/) [38], for which KOBAS (KEGG Orthology Based Annotation System) (http://kobas.cbi.pku.edu.cn) was used to test the statistical enrichment of the target gene candidates identified in the KEGG pathways [39].

\section{Validating the expression of BLS-responsive miRNAs and their target genes by qRT-PCR.}

The expression of 10 target genes was validated by qRT-PCR. Total RNA per sample was extracted with the TaKaRa MiniBEST Universal RNA Extraction Kit (Takara, Tokyo, Japan) and this RNA was then reverse-transcribed to cDNA using the PrimeScript 1st Strand cDNA Synthesis Kit (Takara, Japan). The qRT-PCR was run using the TB Green premix Ex Taq II Kit in a Roche LC480 in order to detect the expression of the target genes. Total small RNA per sample was extracted using RNAiso for Small RNA (Takara, Tokyo, Japan), and this RNA was then reverse-transcribed into cDNA and validated using the Mir$X^{T M}$ miRNA First-Strand Synthesis and TB Green ${ }^{\text {TM }}$ qRT-PCR User Manual (Takara, Japan) in a Roche LC480. The primers used for this are listed in Table 4; here, TC139057 and 5SrRNA served as internal controls. The cycling parameters were $95^{\circ} \mathrm{C}$ for $30 \mathrm{~s}(1 \mathrm{cycle}) ; 95^{\circ} \mathrm{C}$ for $5 \mathrm{~s}, 60^{\circ} \mathrm{C}$ for $30 \mathrm{~s}$ ( 40 cycles); $95^{\circ} \mathrm{C}$ for $5 \mathrm{~s}, 60^{\circ} \mathrm{C}$ for $1 \mathrm{~min}(1 \mathrm{cycle}) ; 40^{\circ} \mathrm{C}$ for $5 \mathrm{~s}$ ( 1 cycle). Gene expression was calculated using the $2^{-\Delta \Delta C t}$ method [40] for which the Ct value is the average of three biological replicates with three technical replicates.

\section{Results}

\section{Difference in BLS resistance between Kunlun14 and Z1141 barley.}

The proportion of total leaf area that was diseased by BLS in KL14 was $12.80 \%$, while it was nearly four times that in $\mathrm{Z1141}$ (47.43\%) (Fig. 1A and B). The proportion of field area (per square meter) diseased by BLS of Kunlun14 was $2.67 \%$, while it was $92.67 \%$ for Z1141, based on field experiments conducted from 2015 to 2017 (Fig. 1C and D). The percentages differed significantly between the two barley cultivars ( $P<$ 0.01), confirming that Kunlun14 is a BLS-resistant cultivar, whereas Z1141 is BLS-susceptible.

\section{Basic statistics of the small RNA data from Tibetan hulless barley libraries.}

To identify BLS-related miRNAs in Tibetan hulless barley, small RNA libraries were constructed for Z1141 with (ZS) and without (ZN) BLS, and Kunlun14 with (KL14S) and without (KL14N) BLS. Totals of $15,141,660,14,167,060,13,591,121$, and $12,992,954$ raw reads were respectively generated from $Z S$, ZN, $\mathrm{KL} 14 \mathrm{~S}$, and KL14N by high-throughput sequencing (Table 1). After removing the adaptors and junk reads, this left corresponding totals of $14,608,407,13,573,623,13,113,086$, and $12,567,992$ clean reads.

Sequences within the range of 18-30 nt were then further analyzed. The length distributions from the libraries tested were similar overall, with most of the small RNAs being 19 to $24 \mathrm{nt}$ in length. Among them, small RNAs with $20 \mathrm{nt}$ of ZN were the most abundant, comprising $16.14 \%$ of the total reads (Fig. 2).

Identification of conserved and novel miRNAs in Tibetan hulless barley. 
To identify conserved miRNAs from the small RNA libraries tested, reads with lengths of 18-30 nt were mapped onto $H$. vulgare-conserved miRNAs in the miRBase 22. This detected 36 conserved miRNAs in all the libraries tested, which belonged to 29 miRNA families (Table 2). Different families thus had different numbers of miRNAs. The family of hvu-miR5049 had the most members, with five miRNAs. The expression levels of different miRNAs differed significantly, with read numbers ranging from zero to several thousand. The 10 most highly-expressed miRNAs were hvu-miR159b, hvu-miR156a, hvumiR5048a, hvu-miR5051, hvu-miR168, hvu-miR171, hvu-miR397a, hvu-miR171, hvu-miR5049e, and hvumiR6198; their accumulated reads accounted for $99.50 \%$ of all total conserved reads. Among them, hvumiR159b had the highest number of reads, with an average of 4476 per library, amounting to $68.81 \%$ of the total conserved reads (Fig. 3A).

After prediction, a total of 56 novel miRNAs were obtained (Table 3). The 10 most highly expressed novel miRNAs were hvu-novel-9, novel-1, hvu-novel-2, hvu-novel-16, hvu-novel-11, hvu-novel-46, hvu-novel-52, hvu-novel-7, hvu-novel-91, and hvu- novel-18, and their accumulative reads accounted for $96.21 \%$ of the total conserved reads. Among them, hvu-novel-2 had the highest number of reads, with an average of 68.81 reads in each library, which accounted for $34.31 \%$ of the total conserved reads (Fig. 3B).

\section{Identification of miRNAs expressed in two Tibetan hulless barley genotypes infected with BLS.}

Expression profiling analysis revealed that the expression of many miRNAs was modulated by BLS. To identify the differentially expressed miRNAs, Venn diagram analysis was used to predict both conserved and novel miRNAs. There were $28,27,19$, and 22 conserved miRNAs detected in the libraries of KL14N, KL14S, ZN, and ZS, respectively; 12 of these miRNAs were common to the four groups (Fig. 4A; Additional file 1). There were $54,28,50$, and 45 novel miRNAs detected in the libraries of KL14N, KL14S, ZN, and ZS, respectively. Of these, 25 were common to the four groups (Fig. 4B; Additional file 2). A total of 24 miRNAs differed significantly between KL14N vs. KL14S and ZN vs. ZS $(P<0.05)$ (Fig. 4C; Additional file $3)$, of which there were 11 conserved miRNAs and 13 novel miRNAs. The expression levels of 24 miRNAs are displayed in a heatmap (Fig. 5; Additional file 4). Based on their fold-change values, 20 miRNAs (including 10 up-regulated miRNAs and 10 down-regulated miRNAs) were found between KL14S and KL14N (Additional file 5), and eight miRNAs were detected between ZS and ZN (six up-regulated miRNAs and two down-regulated miRNAs; Additional file 5). Ultimately, 24 differentially expressed miRNAs were found, of which 11 were conserved miRNAs (hvu-miR6199, hvu-miR168-5p, hvu-miR6198, hvu-miR5049c, hvu-miR171-5p, hvu-miR156a, hvu-miR6181, hvu-miR397a, hvu-miR6195, hvu-miR168-3p, and hvumiR159b) and 13 were novel miRNAs (hvu-novel-43, hvu-novel-119, hvu-novel-1, hvu-novel-45, hvu-novel20, hvu-novel-111, hvu-novel-52, hvu-novel-46, hvu-novel-79, hvu-novel-94, hvu-novel-9, hvu-novel-11, and hvu-novel-91) (Fig. 4C). The differing miRNA expression patterns emphasized the uniqueness of the various miRNA responses to BLS; hence, this suggests that BLS differentially affects miRNA expression in Tibetan hulless barley cultivars with differing disease resistance.

\section{Target gene prediction and analysis of 24 BLS-responsive miRNAs.}


The psRNATarget server predicted 546 target genes of BLS-responsive miRNAs in Tibetan hulless barley that were identifiable for the 24 miRNAs (Additional file 6). Predicted target genes were classified and enriched via gene ontology (GO) and KEGG analyses. GO was used to describe the functional categories of the annotated unigenes and classify the transcripts with known annotated proteins. $P \leq 0.05$ was used as the threshold to determine significantly enriched GO terms. GO classification based on sequence homology revealed that 427 out of all the assembled genes were categorized (Additional file 7). The top 30 enriched GO terms are indicated in Fig. 6, which shows that the target genes of the BLS-responsive miRNAs were included in all three categories: biological process, cellular component, and molecular function. Specifically, the two most enriched categories were heterocyclic compound binding (207 genes) and organic cyclic compound binding (207 genes). Also, a term of copper ion binding (G0:0005507) was significantly enriched in 14 genes, most of which were laccase gene family members (Additional file 8). This indicated that laccase genes may play an important role in the response of Tibetan hulless barley to BLS infection.

A pathway-based analysis was performed using the KEGG pathway database to further elucidate the biological functions and interactions of the gene. We analyzed 546 target genes predicted by the KEGG database. A total of 75 genes were assigned to 64 pathways (Additional file 9). The three main pathways with the highest number of genes were glycerophospholipid metabolism, amino sugar and nucleotide sugar metabolism, and starch and sucrose metabolism. Top 20 enriched KEGG pathways are indicated in Fig. 7, one pathway of which was plant-pathogen interaction, which included four genes (Gene ID: HORVU4Hr1G051540, HORVU3Hr1G079210, HORVU7Hr1G008170, and HORVU7Hr1G055790). These annotations provide valuable information for investigating the disease-resistant pathway involved in BLS.

Transcriptome analysis showed that a total of 131 genes were significantly changed in Kunlun 14 and Z1141 under BLS infection (Additional file 10). Among these genes, we concentrated on the functional genes and transcription factors possibly related to disease-resistant traits according to conserved domain analysis (http://pfam.xfam.org/search) and gene annotation at Swiss-Prot (http://www.gpmaw.com/html/swiss-prot.html), Uniprot (https://www.uniprot.org/), and NCBI (https://www.uniprot.org/). Additionally, considering the negative regulatory relationship between miRNA and target genes and that the relative transcript levels were greater than 20 in one library of KL14N, KL14S, ZN, or ZS (according to the results of Additional file 10), we found seven functional genes and three transcription factors associated with disease resistance. The reference genome sequence information of the candidate genes is shown in Additional file 11. Two members of the P450 genes (Gene ID: HORVU5Hr1G096940 and HORVU5Hr1G096950) were found. Three members of the WRKY transcription factor genes (Gene ID: HORVU1Hr1G090190, HORVU4Hr1G011590, and HORVU7Hr1G083270) were found. RGA gene (Gene ID: HORVU4Hr1G027270), LIN (Gene ID: HORVU2Hr1G104640), SAM (Gene ID: HORVU3Hr1G067760), PSD (Gene ID: HORVU4Hr1G088470), and NDB (Gene ID: HORVU7Hr1G073050) were found. The miRNAs of these target genes were hvu-miR168-3p, hvu-miR156a, hvu-miR171-5p, hvu-miR159b, hvu-novel-91, hvu-novel-46, hvu-novel-52, and hvu-novel-11.

Expression levels of candidate miRNAs and their target genes in the barley response to BLS. 
To validate the differential expression patterns of the BLS-responsive miRNAs, eight miRNAs and 10 target genes were randomly selected and their expression patterns were analyzed by quantitative realtime PCR (qRT-PCR) (Fig. 8). It was observed that the expression patterns of eight miRNAs were correlated with results obtained in the small RNA sequencing (RNA-Seq) study (Additional file 4). We also validated the expression of 10 target genes identified as BLS-responsive in this study. It was observed that the expression patterns of 10 target genes were correlated with results obtained in the RNA-Seq study (Additional file 4). Comparison of the expression patterns of miRNAs with their respective target genes showed that the steady-state levels of the miRNA transcripts were inversely related to the expression patterns of their target genes. For instance, the expression of the target gene (Gene ID:

HORVU4Hr1G027270) was observed to be downregulated in contrast to the expression of the target miR168-3p, which was upregulated in the leaves after infection with BLS (Fig. 8A). On the contrary, the expression of the target genes (Gene ID: HORVU3Hr1G067760, HORVU4Hr1G088470, HORVU1Hr1G090190, HORVU7Hr1G083270, HORVU4Hr1G011590, HORVU5Hr1G096940, HORVU5Hr1G096950, and HORVU7Hr1G073050) was observed to be upregulated in contrast to the expression of their target miRNAs (hvu-miR171-5p, hvu-miR159b, hvu-novel-91, hvu-novel-46, hvu-novel52 , and hvu-novel-11) that were downregulated in the leaves after infection with BLS (Fig. 8C-J).

\section{Discussion}

Increasing numbers of miRNAs are being annotated in various plant species [41, 42]; however, few have been reported for barley $[43,44]$. miRNAs played important roles in the process of plant resistance to disease [18]. In order to better understand the roles of miRNAs in the process of disease resistance, we aimed both to computationally identify different Tibetan hulless barley miRNAs and measure the expression of miRNAs before and after infection with BLS. After infection with BLS, a total of 24 differentially expressed miRNAs were identified, and many of their target genes, such as transcription factor WRKY, NBS-LRR resistance protein, and Cytochrome P450 were found to play important roles in plant growth, development and disease-resistant. Regarding the identification of Tibetan hulless barley miRNAs, 10 known miRNA families (hvu-miR156, hvu-miR168, hvu-miR6198, hvu-miR159, hvu-miR397, hvu-miR171, hvu-miR6195, hvu-miR5049, hvu-miR6199, and hvu-miR6181) and 13 novel miRNAs (hvunovel-1, hvu-novel-11, hvu-novel-43, hvu-novel-45, hvu-novel-46, hvu-novel-52, hvu-novel-79, hvu-novel-91, hvu-novel-111, hvu-novel-119, hvu-novel-9, hvu-novel-20, and hvu-novel-94) differed significantly before and after infection with BLS, which was inferred to be related to the resistance of Tibetan hulless barley to BLS. Many of the 10 identified known miRNAs were known for their reported roles in responses to plant disease and other biotic and abiotic stresses [26].

The miR168 of barley was first isolated in 2010 [45], and it was later isolated from rice [46], barley [47], and cucumber [48] under drought, cadmium, aluminum, and salt stress. A promoter analysis using transgenic $A$. thaliana plants revealed the transcriptional activation of miR168 by fungal elicitors [49], which was upregulated to modulate leaf morphology and plant growth, signified its positive role during leaf curl virus in tomato [26]. In this study, the predicted target gene of hvu-miR168-3p was IBR3 (Gene ID: HORVU4Hr1G027270), which was located on chromosome 4H and annotated to encode an acyl-CoA 
dehydrogenase of $A$. thaliana in Swiss-Prot. In NCBI, HORVU4Hr1G027270 was annotated to encode a clone HV_Mba184-F11 of $H$. vulgare (GenBank accession no. AC256280.1) and LZ-NBS-LRR class RGA of Aegilops tauschii (GenBank accession no. AF446141.1). Nucleotide sequence consistency of them was $90.61 \%$ and $78.98 \%$, respectively. Plants evolved a complex innate immune system that recognized specific pathogens via a specific group of gene products called resistance $(\mathrm{R})$ proteins [50]. RGAs were involved in plant disease resistance [51, 52]. Thus, $R G A$ appears to be crucial to the response of plant disease resistance. In this study, the expression of miR168-3p and its target gene HORVU4Hr1G027270 had opposite trends in the leaves after infection with BLS, which suggested that HORVU4Hr1G027270 might be regulated by hvu-miR168-3p during BLS-resistant of Tibetan hulless barley.

Kantar et al. suggested that miRNA156a in barley leaves responded to dehydration stress and played an important role in stress adaptation [45] (Kantar et al. 2010). miR156a targeted the squamosa promoterbinding protein-like genes, which played an important role in plant growth, development and abiotic stress [53]. In citrus, the overexpression of miR156a or knockout of its target genes SPL3 and SPL 14 significantly increased the activity of wild kumquat callus [54]. In this study, the predicted target gene of hvu-miR156a was LIN (Gene ID: HORVU2Hr1G104640), which was located on chromosome $2 \mathrm{H}$ and annotated to encode an E3 ubiquitin-protein ligase of Medicago truncatula in Swiss-Prot. In NCBI, HORVU2Hr1G104640 was annotated to encode a predicted protein (NIASHv2044K07) of H. vulgare (GenBank accession no. AK366598.1), an E3 ubiquitin-protein ligase LIN-2 of Zea mays, and an E3 ubiquitin-protein ligase LIN-1 of Brachypodium distachyon (GenBank accession no. XM_010242010.3). Nucleotide sequence consistency of them was $99.95 \%, 84.88 \%$, and $78.67 \%$, respectively. In legumes, $L I N$ controlled early infection by rhizobia and worked exclusively during rhizobial colonization [55]. In this study, the expression of hvu-miR156a and its target gene HORVU2Hr1G104640 had opposite trends, which suggested that HORVU2Hr1G104640 might be regulated by hvu-miR156a during BLS-resistant of Tibetan hulless barley.

miR171 was reported in pear and tomato and proved to vital for plant growth and development $[56,57]$. In barley, overexpressing miR171 would lead to phase transitions and floral meristem determinacy [58]. In Arabidopsis, the expression of miR171 increased but the target gene AtSCL6-II decreased when both miR171 and AtSCL6-II were co-express transiently [59]. In addition, another study showed that miR171 overexpression altered the transition from vegetative to reproductive stage by activating the miR156 pathway [59]. Thus, the relationship between miR156 and miR171 in the regulation of BLS remains to be further studied. In this study, the predicted target gene of hvu-miR171-5p was DDB_G0268948 (Gene ID: HORVU3Hr1G067760), which was located on chromosome $3 \mathrm{H}$ and annotated to encode a methyltransferase of Dictyostelium discoideum in Swiss-Prot. In NCBI, HORVU3Hr1G067760 was annotated to encode a predicted protein (NIASHv2113006) of $H$. vulgare (GenBank accession no. AK370649.1) and an S-adenosylmethionine-dependent methyltransferase of Aegilops speltoides (GenBank accession no. FJ236274.1) and Triticum urartu (GenBank accession no.FJ236273.1). Nucleotide sequence consistency of them was $99.73 \%, 93.76 \%$, and $94.11 \%$, respectively. S-adenosyl methionine (SAM), a widespread biological cofactor which was found in all aspects of life where it played very important roles in the transfer of methyl groups to DNA, proteins and small-molecule secondary 
metabolites [60]. The methylation process played important roles in various disease processes and industrial chemical processing [60]. SAM-dependent methylation affected mating through altering the pheromone signaling pathway of the maize smut pathogens (Ustilago maydis) [61]. The second target gene of miR171 was HORVU4Hr1G088470, which was located on chromosome $4 \mathrm{H}$ and annotated to encode a phosphatidylserine decarboxylase proenzyme 1 of Oryza sativa L. subsp. japonica in SwissProt. In NCBI, HORVU4Hr1G088470 was annotated to encode a BAC 615K1 of $H$. vulgare (GenBank accession no. AY485643.1) and a phosphatidylserine decarboxylase (PSD) of Triticum monococcum (GenBank accession no. AY485644.1). Nucleotide sequence consistency of them was $100 \%$ and $94.44 \%$, respectively. PSD activity in oat (Avena sativa) root cells was involved in the changes of phospholipid composition of cell membrane during domestication under drought stress [62]. In this study, the expression of hvu-miR171 and its target genes HORVU3Hr1G067760 and HORVU4Hr1G088470 had opposite trends in the leaves after infection with BLS, which suggested that HORVU3Hr1G067760 and HORVU4Hr1G088470 might be regulated by hvu-miR171-5p during BLS-resistant of Tibetan hulless barley.

In this study, the targets of three miRNAs (hvu-novel-91, hvu-novel-46 and hvu-miR159b) were WRKYs. WRKY proteins was a large family of transcription factors in plants, which was involved in the regulation of plant development, aging and stress resistance $[63,64]$. In rice, WRKY genes were involved in the resistance of the rice blast fungus Pyricularia oryzae [65]. Yokotani et al. showed that an OsWRKY24 gene was upregulated in response to rice blast [66]. In Arabidopsis, the overexpression of WRKY45 or WRKY1 enhanced disease resistance and drought tolerance [44, 67]. In wheat, the overexpression of WRKY45 enhanced disease resistance to multiple fungi [68]. In Swiss-Prot, the target of hvu-novel-91 was a WRKY (Gene ID: HORVU7Hr1G083270), which was located on chromosome 7H and annotated to encode a WRKY46 transcription factor of $A$. thaliana. The target of hvu-novel-46 was a WRKY (Gene ID: HORVU4Hr1G011590), which was located on chromosome 4H and annotated to encode a WRKY1 transcription factor of Zea mays. Furthermore, the target of hvu-miR159b was also a WRKY (Gene ID: HORVU1Hr1G090190), which was located on chromosome 1H and annotated to encode a WRKY53 transcription factor of $A$. thaliana. In NCBI, HORVU7Hr1G083270 was annotated to encode WRKY transcription factor 146 in Triticum (GenBank accession no. MF770640.1), WRKY transcription factor 70 in Aegilops (GenBank accession no. XM_020292626.1), and WRKY transcription factor 11 in Aegilops (GenBank accession no. EU665440.1), but a predicted protein in $H$. vulgare (AK360029.1). The nucleotide sequence consistency of these was $94.83 \%, 94.83 \%, 94.83 \%$, and $87.96 \%$, respectively.

HORVU4Hr1G011590 was annotated to encode four predicted proteins (NIASHv2089N03, NIASHv1011A24, NIASHv3105H03, and FLbaf169m16) in H. vulgare (GenBank accession no. AK369358.1, AK354776.1, AK375802.1, and AK248640.1) and WRKY transcription factor 1 in Aegilops (GenBank accession no. XM_020293740.1), the nucleotide sequence consistency of which was $99.09 \%$, $98.47 \%, 98.77 \%, 98.77 \%$, and $89.94 \%$, respectively. HORVU1Hr1G090190 was annotated to encode WRKY transcription factor 1 in H. vulgare (GenBank accession no. DQ863110.1) and WRKY transcription factor 55 in Aegilops (GenBank accession no. XM_020341128.1). Nucleotide sequence consistency of them was $100.00 \%$ and $87.077 \%$, respectively. Research showed that miR 159 played an important role in 
disease symptom induction by a severe strain of Cucumber mosaic viru [69]. The inhibition of Fny-CMVinduced symptoms in Arabidopsis containing mutant alleles for the targets MYB33 and MYB65 of miR159ab confirmed the importance of miR159 in pathogenesis [69]. Phukan et al. showed WRKY included the WRKYGQK motif followed by a $\mathrm{C} \times 45 \mathrm{C} \times 2223 \mathrm{HxH}$ or $\mathrm{Cx} 7 \mathrm{C} \times 23 \mathrm{HxC}$ zinc-finger motif [70]. In this study, the conserved domain results revealed that HORVU7Hr1G083270, HORVU4Hr1G011590, and HORVU1Hr1G090190 possess WRKY DNA -binding domains that belong to WRKY transcription factors. Most of the WRKY genes studied were found to be involved in the disease-resistant of plants. In apple, for example, MdWRKY26 can induce eight pathogenesis-related (PR) genes to enhance host resistance to leaf spot disease [64]; MdWRKY31 regulates plant resistance to Botryosphaeria dothidea through the salicylic acid signaling pathway by interacting with MdHIR4 [71]. In Arabidopsis, the overexpression of AtWRKY33 accelerated and intensified resistance to necrotrophic fungi [72]. These findings strongly suggested that WRKY functions critically in plant resistance to fungal disease. In our study, the expression of hvu-novel-91 was downregulated, while the expression of WRKYs was upregulated in the leaves after infection with BLS. The expression of miRNAs and their target genes had opposite trends in the leaves after infection with BLS, which suggested that HORVU7Hr1G083270, HORVU4Hr1G011590, and HORVU1Hr1G090190 might be regulated by hvu-novel-91, hvu-novel-46m, and hvu-miR159b during BLS-resistant of Tibetan hulless barley.

In Swiss-Prot, the target genes of hvu-novel-52 included CYP81D11a (Gene ID: HORVU5Hr1G096940) and CYP81D11b (Gene ID: HORVU5Hr1G096950), which were located on chromosome 5H and annotated to encode Cytochrome P450 (CYP450) in A. thaliana. In NCBI, HORVU5Hr1G096940 was annotated to encode a CYP450 in Triticum (GenBank accession no. KJ541960.1), a CYP81B1v3 in Lolium rigidum (GenBank accession no. AB159039.1), a CYP81A22 in Echinochloa phyllopogon (GenBank accession no. AB872310.1), and two predicted proteins in H. vulgare (AK363181.1 and AK375492.1). Nucleotide sequence consistency of them was $89.44 \%, 89.74 \%, 83.92 \%, 99.90 \%$, and $95.65 \%$, respectively. HORVU5Hr1G096950 was annotated to encode two predicted proteins (NIASHv3095M21 and NIASHv2013E24) in H. vulgare (GenBank accession no. AK375492.1 and AK363181.1), a CYP450 in Triticum (GenBank accession no. KJ541960.1), a CYP81B1v3 in L. rigidum (GenBank accession no. AB159039.1), and a CYP81A22 gene in Echinochloa phyllopogon (GenBank accession no. AB872310.1). Nucleotide sequence consistency of them was $99.39 \%, 95.34 \%, 93.75 \%, 89.21 \%$, and $82.38 \%$, respectively. Additionally, the conserved domain results showed that HORVU5Hr1G096940 and HORVU5Hr1G096950, possessing cytochrome P450 domains, belong to the CYP450 family. In rice, a CYP-like protein encoded by Os08g01480 helps plants to resist abiotic stresses [73]. In Arabidopsis, the enzymes encoded by CYP94B3 and CYP94C1 could promote the decomposition and inactivation of the plant hormone jasmonyl isoleucine complex, which affects the jasmonic acid metabolism pathway and thus affects the expression of stress-resistant genes [74]. In this study, the expression of hvu-novel-52 was downregulated, while the expression of HORVU5Hr1G096940 and HORVU5Hr1G096950 was upregulated in the leaves after infection with BLS, which suggested that HORVU5Hr1G096940 and HORVU5Hr1G096950 might be involved in the BLS disease process via the activity of hvu-novel-52 during BLS-resistant of Tibetan hulless barley. 
Hvu-novel-11 targeted HORVU7Hr1G073050, which was located on chromosome 7H and annotated to encode the external alternative $\mathrm{NAD}(\mathrm{P}) \mathrm{H}$-ubiquinone oxidoreductase $\mathrm{B} 3$ (NDB3) protein of in $A$. thaliana. In NCBI, HORVU7Hr1G073050 was annotated to a predicted protein (NIASHv2009M23) of H. vulgare (GenBank accession no. AK362739.1) and an external alternative NAD(P)H-ubiquinone oxidoreductase B2 (NDB2) of Aegilops (GenBank accession no. XM_020292911.1). Nucleotide sequence consistency of them was $99.80 \%$ and $93.42 \%$, respectively. A series of treatment groups representing specific AOX and $\mathrm{NDH}$ genes were identified in response to mitochondrial inhibition, plastid inhibition and other abiotic stresses [75]. In this study, the expression of hvu-novel-11 was downregulated, while that of HORVU7Hr1G073050 was upregulated in the leaves after infection with BLS, and the expression of KL14S was significantly higher than that of ZS. This result suggested that HORVU7Hr1G073050 might be involved in the BLS disease process via the activity of hvu-novel-11 during BLS-resistant of Tibetan hulless barley.

\section{Conclusion}

In summary, 4 known miRNAs and 4 novel miRNAs were revealed in this empirical study, the potential roles of which in BLS resistance might involve the regulation of downstream target genes. The expression levels of 8 miRNAs and 10 target genes were tested, suggesting that these miRNAs and their target genes jointly played a vital role in the defense response of Tibetan hulless barley to BLS, although their relationship in the process of resistance to BLS requires further investigation and elucidation. Fourteen laccase genes from GO analysis and four plant-pathogen interaction genes from the KEGG analysis were deleted because the expression of these genes did not change significantly in the result of RNA-Seq; their function requires further study in the future. This study may improve our understanding of miRNA regulation in the response of Tibetan hulless barley to BLS infection in crop fields.

\section{Abbreviations}

QTL: quantitative trait locus; BLS: purple seed coat color; miRNA: microRNA; GO: Gene Ontology; KEGG: Kyoto Encyclopedia of Genes and Genomes; PDA: potato dextrose agar; TPM: transcripts per million; KOBAS: KEGG Orthology Based Annotation System; KL14N: 'Kunlun14' without barley leaf stripe; KL14S: 'Kunlun14' with barley leaf stripe; ZN: 'Z1141' without barley leaf stripe; ZS: 'Z1141' with barley leaf stripe; RNA-Seq: RNA sequencing; SD: standard deviation; qRT-PCR: quantitative real-time PCR.

\section{Declarations}

\section{Funding}

This research was funded by the Natural Science Foundation of China (31660388; 31960427), the Project of Qinghai Science and Technology Department (2019-ZJ-7075), and the Agriculture Research System of China (CARS-05). 
Acknowledgements

We would like to thank Novogene (https://en.novogene.com) for the practical conduction of the Small RNA sequencing and Accdon (www.accdon.com) for editing this manuscript. We are grateful to the reviewers for their valuable comments.

\section{Availability of data and material}

The data supporting the findings of this study are available within the article.

\section{Authors' contributions}

$\mathrm{XHY}$ and WY designed the research protocol, analyzed the data, carried out the experiment, and drafted the manuscript. YHY, and YXB assisted in the data analysis, supervised the protocol and prepared the manuscript. KLW and QYM finally approved the version to be published. All authors read and approved the final manuscript.

\section{Ethics approval and consent to participate}

Not applicable.

\section{Consent for publication}

Not applicable.

\section{Competing interests}

The authors declare that they have no competing interests.

\section{Publisher's Note}

Springer Nature remains neutral with regard to jurisdictional claims in institutional affiliations and published maps.

\section{References}

1. Yao XH, Wu KL, Yao YH, Bai YX, Ye J, Chi DZ. Construction of a high-density genetic map: genotyping by sequencing (GBS) to map purple seed coat color (Psc) in hulless barley. Hereditas. 2018;155:37. https://doi.org/10.1186/s41065-018-0072-6.

2. Zeng XQ, Guo Y, Xu QJ, Mascher M, Guo GG, Li SC,et al. Origin and evolution of qingke barley in Tibet Nat Commun. 2018;9:5433. https://doi.org/10.1038/s41467-018-07920-5.

3. Li YL, Long CL, Kato KJ, Yang CY, Sato K. Indigenous knowledge and traditional conservation of hulless barley (Hordeum vulgare) germplasm resources in the Tibetan communities of Shangri-la, 
Yunnan,SWChina.Genet Resour Crop Evol. 2011;58:645-655. https://doi.org/10.1007/s10722-0109604-2.

4. Wu KL, YaoXH, Yao YH, Chi DZ, Feng Z. Analysis of the relationship between Wx gene polymorphisms and amylose content in hulless barley. Czech J. Genet. Plant Breed. 2017;53:144152. https://doi.org/10.17221/105/2016-CJGPB.

5. Si E, Meng YX, Ma XL, Li BC, Wang JC, Ren PR, et al. Development and characterization of microsatellite markers based on whole-genome sequences and pathogenicity differentiation of Pyrenophora graminea, the causative agent of barley leaf stripe. Eur J Plant Pathol. 2019;154:227241. https://doi.org/10.1007/s10658-018-01651-5.

6. Arabi MIE, Jawhar M, Al-Safadi B, Mirali N. Yield responses of barley to leaf stripe (Pyrenophora graminea) under experimental conditions in southern Syria. J Phytopathol. 2004;152:519-523. https://doi.org/10.1111/j.1439-0434.2004.00891.x

7. Li DH, Guo HQ, Ma L, Ma DF, Wang FT, Feng J, et al. Genetic diversity analysis of Pyrenophora graminea populations in China, the causal organism of barley leaf stripe by using AFLP method. Plant Prot. 2016;42:71-76.

8. Arru L, Francia E,Pecchioni N. Isolate-specific QTLs of resistance to leaf stripe (Pyrenophora graminea) in the 'Steptoe' × 'Morex' spring barley cross. Theor Appl Genet. 2003;106:668-675. https://doi.org/10.1007/s00122-002-1115-x.

9. Biselli C, Urso S, Tacconi G, Steuernagel B, Schulte D, Gianinetti A, et al. Haplotype variability and identification of new functional alleles at the Rdg2a leaf stripe resistance gene locus. Theor Appl Genet. 2013;126:1575-1586. https://doi.org/10.1007/s00122-013-2075-z.

10. Tacconi G, Cattivelli L, Faccini N, Pecchioni N, Stanca AM,Valé G. Identification and mapping of a new leaf stripe resistance gene in barley (Hordeum vulgare L.). Theor Appl Genet. 2001;102:12861291. https://doi.org/10.1007/s001220000510.

11. Gutiérrez L, Germán S, Pereyra S, Hayes PM, Pérez CA, Capettini F, et al. Multi-environment multi-QTL association mapping identifies disease resistance QTL in barley germplasm from Latin America. Theor Appl Genet. 2015;128:501-516. https://doi.org/10.1007/s00122-014-2448-y.

12. Richardson KL,Vales MI, Kling JG, Mundt CC, Hayes PM. Pyramiding and dissecting disease resistance QTL to barley stripe rust. Theor Appl Genet. 2006;113:485-495. https://doi.org/10.1007/s00122-006-0314-2.

13. Arru L, Niks RE, Lindhout P, Valé G,Francia E, Pecchioni N. Genomic regions determining resistance to leaf stripe (Pyrenophoragraminea) in barley. Genome. 2002;45:460-466. https://doi.org/10.1139/g02-004.

14. Biselli C, Bulgarelli D, Collins NC, Schulze-Lefert P, Stanca AM, Cattivelli L.The CC-NB-LRR-type Rdg2a Resistance Gene Evolved Through Recombination and Confers Immunity to the Seed-Borne Barley Leaf Stripe Pathogen in the Absence of Hypersensitive Cell Death. In: Zhang G, Li C, Liu X. (eds) Advance in Barley Sciences. Springer, Dordrecht. 2013;217-228. https://doi.org/10.1007/978-94-0074682-4_19. 
15. Biselli C, Urso S, Bernardo L, Tondelli A, Tacconi G, Martino V, et al. Identification and mapping of the leaf stripe resistance gene Rdg1a in Hordeum spontaneum.Theor Appl Genet. 2010;120:1207-1218. https://doi.org/10.1007/s00122-009-1248-2.

16. BulgarelliD, Biselli C, Collins NC, Consonni G, Stanca AM, Schulze-Lefert P, et al.The CC-NB-LRR-type Rdg2a Resistance Gene Evolved Through Recombination and Confers Immunity to the Seed-Borne Barley Leaf Stripe Pathogen in the Absence of Hypersensitive Cell Death.In:Zhang G,Li C,Liu X. (eds) Advance in Barley Sciences.Springer,Dordrecht. 2010;5:e12599. https://doi.org/10.1007/978-94-0074682-4_19.

17. Haegi A, Bonardi V, Dall'Aglio E, Glissant D, Tumino G, Collins NC, et al. Histological and molecular analysis of Rdg2a barley resistance to leaf stripe. Mol Plant Pathol. 2008;9:463-478. https://doi.org/10.1111/j.1364-3703.2008.00479.x.

18. Chinen M. Diverse functions of nuclear non-coding RNAs in eukaryotic gene expression. Front Biosci. 2012;17(4): 1402-1417. https://doi.org/10.2741/3994.

19. Rawat N, Kiran SP, Du D, Gmitter FG, Jr, Deng Z. Comprehensive meta-analysis, co-expression, and miRNA nested network analysis identifies gene candidates in citrus against Huanglongbing disease. BMC Plant Biol. 2015;15:184. https://doi.org/10.1186/s12870-015-0568-4.

20. Shivaraj SM, Jain A, Singh A. Highly preserved roles of Brassica MIR172 in polyploid Brassicas: ectopic expression of variants of Brassica MIR172 accelerates floral transition. Mol Genet Genomics. 2018;293:1121-1138. https://doi.org/10.1007/s00438-018-1444-3.

21. Tian WJ, Ge YY, Liu XY, Dou GM, Ma YC. Identification and characterization of Populus microRNAs in response to plant growth-promoting endophytic Streptomyces sp. SSD49. World J Microbiol Biotechnol. 2019; 35:97. https://doi.org/10.1007/s11274-019-2671-4.

22. Tu MX, Wang XH, Huang LH, Guo RR, Zhang HJ, Cai JS, et al. Expression of a grape bZIP transcription factor, VqbZIP39, in transgenic Arabidopsis thaliana confers tolerance of multiple abiotic stresses. Plant Cell Tissue Organ Cult. 2016;125:537-551. https://doi.org/10.1007/s11240016-0969-6.

23. Zhu JF, L, WF, Yang WH, Qi LW, Han SY. Identification of microRNAs in Caragana intermedia by highthroughput sequencing and expression analysis of 12 microRNAs and their targets under salt stress. Plant Cell Rep.2013;32:1339-1349. https://doi.org/10.1007/s00299-013-1446-x.

24. Parry G, Calderon-Villalobos LI, Prigge M, Peret B, Dharmasiri S, Itoh H, et al. Complex regulation of the TIR1/AFB family of auxin receptors. Proc Natl Acad Sci U. S. A. 2009;106:22540-22545. https://doi.org/10.1073/pnas.0911967106.

25. Kis A, Tholt G, Ivanics M, Várallyay É, Jenes B, Havelda Z. Polycistronic artificial miRNA-mediated resistance to Wheat dwarf virus in barley is highly efficient at low temperature. Mol Plant Pathol. 2016;17:427-437. https://doi.org/10.1111/mpp.12291.

26. Kumar G, Singh A, Vinutha T, Kumar S, Kumar RR., Praveen S. Possible role of miRNAs and their targets, in modulating leaf morphology and plant growth during leaf curl virus infection in tomato. Indian Journal of Plant Physiology. 2017;22:608-615. https://doi.org/10.1007/s40502-017-0345-5. 
27. Yin Z, Li Y, Han X, Shen F. Genome-wide profiling of miRNAs and other small non-coding RNAs in the Verticillium dahliaeinoculated cotton roots. PLoS One. 2012;7:e35765.

https://doi.org/10.1371/journal.pone.0035765.

28. Wu J, Yang Z, Wang Y, Zheng L, Ye R, Ji Y, et al. Viral-inducible Argonaute18 confers broad-spectrum virus resistance in rice by sequestering a host microRNA. eLife. 2015;4:e05733. https://doi.org/10.7554/eLife.05733.

29. Langmead B, Trapnell C, Pop M, Salzberg SL. Ultrafast and memory-efficient alignment of short DNA sequences to the human genome. Genome Biol. 2009;10:R25. https://doi.org/10.1186/gb-2009-10-3r25.

30. Mascher M, Gundlach H, Himmelbach A, Beier S, Twardziok SO, Wicker T, et al. A chromosome conformation capture ordered sequence of the barley genome. Nature. 2017;544:427-433. https://doi.org/10.1186/gb-2009-10-3-r25.

31. Friedländer MR, Mackowiak SD, Li N, Chen W, Rajewsky N. miRDeep2 accurately identifies known and hundreds of novel microRNA genes in seven animal clades. Nucleic Acids Res. 2012;40:37-52. https://doi.org/10.1093/nar/gkr688.

32. Moxon S, Schwach F, Dalmay T, MacLean D, Studholme DJ, Moulton V. A toolkit for analysing largescale plant small RNA datasets. Bioinformatics. 2008;24:2252-2253.

https://doi.org/10.1093/bioinformatics/btn428.

33. Wen M, Shen Y, Shi S, Tang T. miREvo: an integrative microRNA evolutionary analysis platform for next-generation sequencing experiments. BMC Bioinformatics. 2012;13:140. https://doi.org/10.1186/1471-2105-13-140.

34. Li B, Ruotti V, Stewart RM, Thomson JA, Dewey CN. RNA-Seq gene expression estimation with read mapping uncertainty. Bioinformatics. 2010;26:493-500. https://doi.org/10.1093/bioinformatics/btp692.

35. Qiu L, Chen R, Fan Y, Huang X, Luo H, Xiong F, et al. Integrated mRNA and small RNA sequencing reveals microRNA regulatory network associated with internode elongation in sugarcane (Saccharum officinarum L.). BMC Genomics. 2019;20:817. https://doi.org/10.1186/s12864-019-6201-4.

36. Benjamini Y, Hochberg Y. Controlling the false discovery rate: a practical and powerful approach to multiple testing. Journal of the Royal Statistical Society. Series B(Methodological). 1995;57:289-300. https://doi.org/10.2307/2346101.

37. Tripathi RK, Bregitzer P, Singh J. Genome-wide analysis of the SPL/miR156 module and its interaction with the AP2/miR172 unit in barley. Sci Rep 2018;8:7085. https://doi.org/10.1038/s41598-018-25349-0.

38. Liu M, Yu H, Zhao G, Huang Q, Lu Y, Ouyang B. Identification of drought-responsive microRNAs in tomato using high-throughput sequencing. Funct Integr Genomics. 2018;18:67-78. https://doi.org/10.1007/s10142-017-0575-7.

39. Mao X, Cai T, Olyarchuk JG, Wei L. Automated genome annotation and pathway identification using the KEGG Orthology (KO) as a controlled vocabulary. Bioinformatics. 2005;21:3787-3793. 
https://doi.org/10.2307/1592215.

40. Livak KJ, Schmittgen TD. Analysis of relative gene expression data using real-time quantitative PCR and the $2^{-\triangle \Delta C T}$ method. Methods. 2001;25:402-408. https://doi.org/10.1006/meth.2001.1262.

41. Solanki M, Sinha A, Shukla LI. The miR408 expression in scutellum derived somatic embryos of Oryza sativa L. ssp. indica varieties: media and regenerating embryos. Plant Cell Tissue Organ Cult. 2019;138:53-66. https://doi.org/10.1007/s11240-019-01602-w.

42. Zhao CZ, Xia H, Frazier TP, Yao YY, Bi YP, Li AQ, et al. Deep sequencing identifies novel and conserved microRNAs in peanuts (Arachis hypogaea L.). BMC Plant Biol. 2010;10:3. https://doi.org/10.1186/1471-2229-10-3.

43. Lv S, Nie X, Wang L, Du X, Biradar SS, Jia X, et al. Identification and characterization of microRNAs from barley (Hordeum vulgare L.) by high-throughput sequencing. International journal of molecular sciences. 2012;13:2973-2984. https://doi.org/10.3390/ijms13032973.

44. Qiu Y, Yu D. Over-expression of the stress-induced OsWRKY45 enhances disease resistance and drought tolerance in Arabidopsis. Environ Exp Bot. 2009;65:35-47. https://doi.org/10.1016/j.envexpbot.2008.07.002.

45. Kantar M, Unver T, Budak $\mathrm{H}$. Regulation of barley miRNAs upon dehydration stress correlated with target gene expression. Funct Integr Genomics. 2010;10:493-507. https://doi.org/10.1007/s10142010-0181-4.

46. Shin SJ, Ahn H, Jung I, Rhee S, Kim S, Kwon, HB. Novel drought-responsive regulatory coding and non-coding transcripts from Oryza Sativa L. Genes \& Genomics. 2016;38:949-960. https://doi.org/10.1007/s13258-016-0439-x.

47. Kruszka K, Pacak A, Swida-Barteczka A, Stefaniak AK, Kaja E, Sierocka I, et al. Developmentally regulated expression and complex processing of barley pri-microRNAs. BMC Genomics. 2013;14:34. https://doi.org/10.1186/1471-2164-14-34.

48. Li Y, Li C, Bai L, He C, Yu X. MicroRNA and target gene responses to salt stress in grafted cucumber seedlings. Acta Physiol. Plant. 2016;38:42. https://doi.org/10.1007/s11738-016-2070-5.

49. Baldrich P, Kakar K, Siré C, Moreno AB, Berger A, García-Chapa M, et al. Small RNA profiling reveals regulation of Arabidopsis miR168 and heterochromatic siRNA415 in response to fungal elicitors. BMC Genomics. 2014;15:1083. https://doi.org/10.1186/1471-2164-15-1083.

50. Cheng XJ, He B, Chen L, Xiao SQ, Fu J, Chen Y, et al. Transcriptome analysis confers a complex disease resistance network in wild rice Oryza meyeriana against Xanthomonas oryzae pv. oryzae. Scientific. Reports. 2016;6:38215. https://doi.org/10.1038/srep38215.

51. Reddy AC, Venkat S, Singh TH, Aswath C, Reddy KM, Reddy DCL. Isolation, characterization and evolution of NBS-LRR encoding disease-resistance gene analogs in eggplant against bacterial wilt. European Journal of Plant Pathology. 2015;143:417-426. https://doi.org/10.1007/s10658-015-06939.

52. Suraby EJ, Prasath D, Babu KN, Anandaraj M. Identification of resistance gene analogs involved in Phytophthora capsici recognition in black pepper (Piper nigrum L.). J Plant Pathol. 2020;3:1-11. 
https://doi.org/10.1007/s42161-020-00586-3.

53. Xu M, Hu T, Zhao J, Park MY, Earley KW, Wu G, et al. Developmental functions of miR156-regulated squamosa promoter binding protein-like (spl) genes in Arabidopsis thaliana. PLoS Genetis.2016;12:8. https://doi.org/10.1371/journal.pgen.1006263.

54. Long JM, Liu CY, Feng MQ, LiuY, Wu XM,Guo WW. MiR156-SPL modules regulate induction of somatic embryogenesis in citrus callus. Journal of Experimental Botany. 2018;69:2979-2993. https://doi.org/10.1093/jxb/ery132.

55. Kiss E, Oláh B, Kaló P, Morales M, Heckmann AB, Borbola A, et al. LIN, a novel type of U-box/WD40 protein, controls early infection by rhizobia in legumes. Plant Physiol. 2009; 151:1239-1249. https://doi.org/10.1104/pp.109.143933.

56. Jiang S, Chen Q, Zhang Q, Zhang Y, Hao N, Ou C, et al. Pyr-miR171f-targeted PyrSCL6 and PyrSCL22 genes regulate shoot growth by responding to IAA signaling in pear. Tree Genetics\&Genomes. 2018;14:20. https://doi.org/10.1007/s11295-018-1233-5.

57. Kravchik M, Stav R, Belausov E, Arazi T. Functional characterization of microRNA171 family in tomato. Plants. 2019;8:10. https://doi.org/10.3390/plants8010010.

58. Curaba J, Talbot M, Li ZY, Helliwell C. Over-expression of microRNA171 affects phase transitions and floral meristem determinancy in barley. BMC Plant Biology. 2013;13:6. https://doi.org/10.1186/14712229-13-6.

59. Hai BZ, Qiu ZB, He YY, Yuan MM, Li YF. Characterization and primary functional analysis of Pinus densata miR171. Biol. Plant. 2018;62:318-324. https://doi.org/10.1007/s10535-018-0774-7.

60. Struck AW, Thompson ML, Wong LS, Micklefield J. S-adenosyl-methionine-dependent methyltransferases: highly versatile enzymes in biocatalysis, biosynthesis and other biotechnological applications. Chembiochem 2012;13:2642-2655. https://doi.org/10.1002/cbic.201200556.

61. Fischer JA, McCann MP, Snetselaar KM. Methylation is involved in the Ustilago maydis mating response. Fungal Genetis \&Biology. 2001;34:21-35. https://doi.org/10.1006/fgbi.2001.1287.

62. Larsson KE, Nyström B, Liljenberg C. A phosphatidylserine decarboxylase activity in root cells of oat (Avena sativa) is involved in altering membrane phospholipid composition during drought stress acclimation. Plant PhysiolBiochem. 2006;44:211-219. https://doi.org/10.1016/j.plaphy.2006.04.002..

63. Zhang CQ, Xu Y, Lu Y, Yu HX, Gu MH, Liu QQ. The WRKY transcription factor OsWRKY78 regulates stem elongation and seed development in rice. Planta. 2011;234:541-554. https://doi.org/10.1007/s00425-011-1423-y.

64. ZhangQ, LiY, ZhangY, WuC, WangS, HaoL, et al. Md-miR156ab and Md-miR395 target WRKY transcription factors to influence apple resistance to leaf spot disease. Frontiers in Plant ence. 2017;8:526. https://doi.org/10.3389/fpls.2017.00526.

65. Jimmy JL, Babu S. Role of OsWRKY transcription factors in rice disease resistance. Trop. Plant Pathol. 2015;40:355-361. https://doi.org/10.1007/s40858-015-0058-0. 
66. Yokotani N, Shikata M, Ichikawa H, Mitsuda N, Ohme-Takagi M, Minami E. OsWRKY24, a blastdisease responsive transcription factor, positively regulates rice disease resistance. Journal of General Plant Pathology. 2018;84:85-91. https://doi.org/10.1007/s10327-018-0768-5.

67. Cui XW, Zhang Y, QiF Y, Gao J, Chen YW, Zhang CL. Overexpression of a moso bamboo (Phyllostachys edulis) transcription factor gene PheWRKY1 enhances disease resistance in transgenic Arabidopsis thaliana. Botany-botanique. 2013;91:486-494. https://doi.org/10.1139/cjb2012-0219.

68. Bahrini I, Ogawa T, Kobayashi F, Kawahigashi H, Handa H. Overexpression of the pathogen-inducible wheat TaWRKY45 gene confers disease resistance to multiple fungi in transgenic wheat plants. Breed Sci. 2011;61:319-326. https://doi.org/10.1270/jsbbs.61.319.

69. Du Z, Chen A, Chen W, Westwood JH, Baulcombe DC, Carr JP. Using a viral vector to reveal the role of microRNA159 in disease symptom induction by a severe strain of Cucumber mosaic virus. Plant Physiol. 2014;164:1378-1388. https://doi.org/10.1104/pp.113.232090.

70. Phukan UJ, Jeena GS, Shukla RK. WRKY transcription factors: molecular regulation and stress responses in plants. Front. Plant Sci. 2016;7:760. https://doi.org/10.3389/fpls.2016.00760.

71. Zhao XY, Qi CH, Jiang H, Zhong MS, You CX, Li YY, et al. MdHIR4 transcription and translation levels associated with disease in apple are regulated by MdWRKY31. Plant Mol Biol. 2019;101:149-162. https://doi.org/10.1007/s11103-019-00898-8.

72. Zheng Z, Qamar SA, Chen Z, Mengiste T. Arabidopsis WRKY33 transcription factor is required for resistanceto necrotrophic fungal pathogens. Plant Journal. 2006;48:592-605. https://doi.org/10.1111/j.1365-313X.2006.02901.x.

73. Rai A, Singh R, Shirke PA, Tripathi RD, Trivedi PK., Chakrabarty D. Expression of rice CYP450-like gene (Os08g01480) in Arabidopsis modulates regulatory network leading to heavy metal and other abiotic stress tolerance. PLoS One. 2015; 10(9): e0138574. https://doi.org/10.1371/journal.pone.0138574.

74. Koo AJK, Cooke TF, Howe GA. Cytochrome P450 CYP94B3 mediates catabolism and inactivation of the plant hormone jasmonoyl-L-isoleucine. Proc Natl Acad Sci USA. 2011;108:9298-9303. https://doi.org/10.1073/pnas.1103542108.

75. Clifton R, Lister R, Parker KL, Sappl PG, Elhafez D, Millar AH, et al. Stress-induced co-expression of alternative respiratory chain components in Arabidopsis thaliana. Plant Mol Biol. 2005;58:193. https://doi.org/10.1007/s11103-005-5514-7.

\section{Tables}

Table 1. Summary of small RNA sequencing data in four libraries from Tibetan hulless barley 
Type

KL14N KL14S Z

\begin{tabular}{|c|c|c|c|c|c|c|c|}
\hline Count & $\begin{array}{l}\text { Percentage } \\
(\%)\end{array}$ & Count & $\begin{array}{l}\text { Percentage } \\
\text { (\%) }\end{array}$ & Count & $\begin{array}{l}\text { Percentage } \\
(\%)\end{array}$ & Count & $\begin{array}{l}\text { Percentage } \\
(\%)\end{array}$ \\
\hline
\end{tabular}

\begin{tabular}{|c|c|c|c|c|c|c|c|c|}
\hline Raw reads & 12992954 & 100 & 13591121 & 100 & 14167060 & 100 & 15141660 & 100 \\
\hline Clean reads & 12567992 & 96.73 & 13113086 & 96.48 & 13573623 & 95.81 & 14608407 & 96.48 \\
\hline Total sRNA & 8483600 & 65.29 & 7094377 & 52.20 & 7377096 & 52.07 & 8930242 & 58.98 \\
\hline $\begin{array}{l}\text { Mapped } \\
\text { sRNA }\end{array}$ & 7698518 & 59.25 & 6055386 & 44.55 & 6643849 & 46.90 & 6554917 & 43.29 \\
\hline rRNA & 3806241 & 29.29 & 3892847 & 28.64 & 3808842 & 26.89 & 4402208 & 29.07 \\
\hline tRNA & 263883 & 2.03 & 151163 & 1.11 & 147841 & 1.04 & 230688 & 1.52 \\
\hline snRNA & 2060 & 0.02 & 5209 & 0.04 & 1863 & 0.01 & 5210 & 0.03 \\
\hline snoRNA & 6195 & 0.05 & 4168 & 0.03 & 3739 & 0.03 & 4789 & 0.03 \\
\hline miRNA & 22408 & 0.17 & 2057 & 0.02 & 14273 & 0.10 & 5600 & 0.04 \\
\hline Exon & 113098 & 0.87 & 69152 & 0.51 & 72516 & 0.51 & 54421 & 0.36 \\
\hline Intron & 46160 & 0.36 & 5025 & 0.04 & 25199 & 0.18 & 9020 & 0.06 \\
\hline TAS & 26 & 0.00 & 8 & 0.00 & 8 & 0.00 & 2 & 0.00 \\
\hline Repeat & 293923 & 2.26 & 76478 & 0.56 & 195502 & 1.38 & 71723 & 0.47 \\
\hline $\begin{array}{l}\text { Other } \\
\text { ncRNA }\end{array}$ & 1826849 & 14.06 & 1020473 & 7.51 & 1534211 & 10.83 & 1315953 & 8.69 \\
\hline
\end{tabular}

KL14N = 'Kunlun14' without barley leaf stripe; KL14S = 'Kunlun14' with barley leaf stripe; miRNA = microRNA; TAS = ta-siRNA; ZN = 'Z1141' without barley leaf stripe; ZS = 'Z1141' with barley leaf stripe.

Table 2. The conserved miRNAs in Tibetan hulless barley 


\begin{tabular}{|c|c|c|c|c|}
\hline miRNA family & miRNA & Sequence & Length & Accession number \\
\hline $\operatorname{miR} 1120$ & hvu-miR1120 & ACAUUCUUAUAUUAUGGGACGGAG & 24 & MIMAT0018499 \\
\hline $\operatorname{miR} 1436$ & hvu-miR1436 & ACAUUAUGGGACGGAGGGAGU & 21 & MIMAT0018501 \\
\hline $\operatorname{miR} 156$ & hvu-miR156a & UGACAGAAGAGAGUGAGCACA & 21 & MIMAT0018207 \\
\hline $\operatorname{miR} 159$ & hvu-miR159b & UUUGGAUUGAAGGGAGCUCUG & 21 & MIMAT0018209 \\
\hline \multirow[t]{2}{*}{$\operatorname{miR} 168$} & hvu-miR168-3p & GAUCCCGCCUUGCACCAAGUGAAU & 24 & MIMAT0018216 \\
\hline & hvu-miR168-5p & UCGCUUGGUGCAGAUCGGGAC & 21 & MIMAT0018215 \\
\hline \multirow[t]{2}{*}{$\operatorname{miR} 171$} & hvu-miR171-5p & UGUUGGCUCGACUCACUCAGA & 21 & MIMAT0022971 \\
\hline & hvu-miR171-3p & UGAUUGAGCCGUGCCAAUAUC & 21 & MIMAT0018220 \\
\hline \multirow[t]{2}{*}{ miR397 } & hvu-miR397a & CCGUUGAGUGCAGCGUUGAUG & 21 & MIMAT0018224 \\
\hline & hvu-miR397b-3p & AUCAACGCUGCACUCAACGGC & 21 & MIMAT0035814 \\
\hline $\operatorname{miR} 444$ & hvu-miR444b & UGCAGUUGCUGUCUCAAGCUU & 21 & MIMAT0020543 \\
\hline $\operatorname{miR} 5048$ & hvu-miR5048a & UAUUUGCAGGUUUUAGGUCUAA & 22 & MIMAT0020544 \\
\hline \multirow[t]{5}{*}{$\operatorname{miR} 5049$} & hvu-miR5049a & UCCUAAAUACUUGUUGUUGGG & 21 & MIMAT0020545 \\
\hline & hvu-miR5049c & AGACAAUUAUUUUGGGACGGAGG & 23 & MIMAT0024803 \\
\hline & hvu-miR5049d & UACAAUUAUUUAGGAACGGAG & 21 & MIMAT0024813 \\
\hline & hvu-miR5049e & AAUUAUUUAGGUACAGAGGGA & 21 & MIMAT0024826 \\
\hline & hvu-miR5049f & AAUUAAUAUGGAUCGGAGGGA & 21 & MIMAT0024829 \\
\hline miR5051 & hvu-miR5051 & UUUGGCACCUUGAAACUGGGA & 21 & MIMAT0020547 \\
\hline miR5052 & hvu-miR5052 & ACCGGCUGGACGGUAGGCAUA & 21 & MIMAT0020548 \\
\hline $\operatorname{miR} 6177$ & hvu-miR6177 & UACCAUGGACAGAAGGCACUUA & 22 & MIMAT0024800 \\
\hline $\operatorname{miR6180}$ & hvu-miR6180 & AGGGUGGAAGAAAGAGGGCG & 20 & MIMAT0024804 \\
\hline miR6181 & hvu-miR6181 & UGCUCUUCAUGGACUGCGGCGCC & 23 & MIMAT0024805 \\
\hline $\operatorname{miR} 6182$ & hvu-miR6182 & UGAGUGUGUGAUGGAUGGCUUU & 22 & MIMAT0024806 \\
\hline $\operatorname{miR6183}$ & hvu-miR6183 & UGAGCGAGUUGGCUGCAAGUUC & 22 & MIMAT0024807 \\
\hline miR6186 & hvu-miR6186 & CGAGGAAGGCGCUGAGAGAGA & 21 & MIMAT0024810 \\
\hline $\operatorname{miR} 6191$ & hvu-miR6191 & UAGAUUUGUCUAGAUAUGAA & 20 & MIMAT0024816 \\
\hline $\operatorname{miR6194}$ & hvu-miR6194 & UAUGGGGAUCUGACAGACGAG & 21 & MIMAT0024820 \\
\hline $\operatorname{miR} 6195$ & hvu-miR6195 & UGAGUACGUAGUAGGGAUGAG & 21 & MIMAT0024821 \\
\hline
\end{tabular}

Page 22/36 


\begin{tabular}{|ccccc|} 
miR6198 & hvu-miR6198 & GCUCUGUCUUGGAUGGUCAUUC & 22 & MIMAT0024824 \\
miR6199 & hvu-miR6199 & CCACAGAAUUCUCACAGUGAUGG & 23 & MIMAT0024825 \\
miR6200 & hvu-miR6200 & UUUGGCCAACUAGAUCUAUGA & 21 & MIMAT0024827 \\
miR6201 & hvu-miR6201 & UGACCCUGAGGCACUCAUACCG & 22 & MIMAT0024828 \\
miR6202 & hvu-miR6203 & AGACGAUUAAGAAGACCUGCAA & 22 & MIMAT0024831 \\
miR6207 & hvu-miR6207 & UGGACGACCUGGGCGCCGACG & 21 & MIMAT0024835 \\
miR6213 & hvu-miR6213 & ACAGAUUGCUACAGACUGGUC & 21 & MIMAT0024841 \\
miR6214 & hvu-miR6214 & CGACGACGACGAGCACGACA & 20 & MIMAT0024842 \\
\hline
\end{tabular}

$\operatorname{miRNA}=$ microRNA.

Table 3. The novel miRNAs identified in this study of Tibetan hulless barley 


\begin{tabular}{|c|c|c|}
\hline Novel miRNAs & Sequence & Length \\
\hline hvu-novel-1 & UGAAGCUGCCAGCAUGAUCUGC & 22 \\
\hline hvu-novel-100 & UUCACGGGUCUGGACAAUA & 19 \\
\hline hvu-novel-102 & GCGUAUCUAGAUCGUUGAUGAUGU & 24 \\
\hline hvu-novel-107 & AGACGUCUCUCGUGUAGAAAUAGG & 24 \\
\hline hvu-novel-11 & UCAGUGCGAUCCCUCUGGAAU & 21 \\
\hline hvu-novel-111 & AUAAUGUAGGACGUGUUUUGA & 21 \\
\hline hvu-novel-119 & AAAUCGGUGAUCUAGAACUACGCU & 24 \\
\hline hvu-novel-12 & UGACAGAAGAGAGUGAGCAC & 20 \\
\hline hvu-novel-16 & UUCAGGAGAGAUGACACCGACA & 22 \\
\hline hvu-novel-18 & UGCACUGCCUCUUCCCUGGC & 20 \\
\hline hvu-novel-2 & UCCACAGGCUUUCUUGAACUG & 21 \\
\hline hvu-novel-20 & UUCCACAGCUUUCUUGAACUG & 21 \\
\hline hvu-novel-22 & UGGAGAAGCAGGGCACGUGCA & 21 \\
\hline hvu-novel-27 & ACGACUGCGCGUACAUCGGACGGA & 24 \\
\hline hvu-novel-28 & GGAGUGUCUCUGAGAAAACAAG & 22 \\
\hline hvu-novel-30 & GUGUGCUGUAUCGGCUGUUGUUCU & 24 \\
\hline hvu-novel-31 & CCGCGGUAGAACAGAACCCACAUC & 24 \\
\hline hvu-novel-33 & CGUGGCCUACUGACCUGACGCGUC & 24 \\
\hline hvu-novel-34 & ACGAUCUAGAACUACGCGCAUGCC & 24 \\
\hline hvu-novel-37 & GAAUGCAUGCAGUCUACUAGUAGU & 24 \\
\hline hvu-novel-38 & UAGCCAAGGAUGAUUUGCCUGUG & 23 \\
\hline hvu-novel-39 & CGCAUGAAUGGAUCAAUGA & 19 \\
\hline hvu-novel-42 & AUGACGUUGGUGCUUGCAUUUUCC & 24 \\
\hline hvu-novel-43 & AUAAUGUAGGACGUGUUUUGAC & 22 \\
\hline hvu-novel-45 & UUUAGGACAAGUAUUUCCAGACGG & 24 \\
\hline hvu-novel-46 & AAACUGUGGCUGUCAAAUUUUGGC & 24 \\
\hline hvu-novel-48 & AGGAUAUGAUGGCUCGCGGACGCG & 24 \\
\hline hvu-novel-52 & UUCCACAGCUUUCUUGAACUU & 21 \\
\hline
\end{tabular}

Page 24/36 


\begin{tabular}{|c|c|c|}
\hline hvu-novel-55 & GAAGACGUGCCUGGCUGUGUUACC & 24 \\
\hline hvu-novel-56 & AGUGCAACGACUGAGGCUUAGGCG & 24 \\
\hline hvu-novel-57 & UUUCCUGAUUUGUUUUCACCGAGC & 24 \\
\hline hvu-novel-58 & GUUCUUUGCCGUCUGUAGUACGAA & 24 \\
\hline hvu-novel-59 & AGCGACUGUUCCAUAGAUGAAAGC & 24 \\
\hline hvu-novel-60 & UCUGGUAGGCCUGUGUGUACGACC & 24 \\
\hline hvu-novel-61 & AGAAUAUGAGGGCUCGCGGACGCG & 24 \\
\hline hvu-novel-65 & AGCUCGUUUGAUCCUGAUG & 19 \\
\hline hvu-novel-68 & UGAAGCUGCCAGCAUGAUCUAAC & 23 \\
\hline hvu-novel-69 & AGGAUAUGAGGGUUCAUGGACGUG & 24 \\
\hline hvu-novel-7 & UGAAGCUGCCAGCAUGAUCUGA & 22 \\
\hline hvu-novel-70 & GUUCUAUUGGACUGUCUGACCAGU & 24 \\
\hline hvu-novel-72 & AAUUGACGAUCUAGAUAUAUGUGC & 24 \\
\hline hvu-novel-73 & GUGUAGCUCCCGGGUGAUUUGAUC & 24 \\
\hline hvu-novel-74 & AUGAGAACUUUGAAGACU & 18 \\
\hline hvu-novel-76 & AUCAGGAGAGAUGACACCGGC & 21 \\
\hline hvu-novel-79 & AAGGUCUCUGACGUCUGGUACGGG & 24 \\
\hline hvu-novel-80 & AUUAGACUGUGUGACGCCUUAGA & 23 \\
\hline hvu-novel-81 & CAACUAUUUCCGGACUGAGGGAGU & 24 \\
\hline hvu-novel-83 & UGACUGAAUUUUGCACUA & 18 \\
\hline hvu-novel-86 & CAAUUAACGACCUAGAACUACGCG & 24 \\
\hline hvu-novel-87 & AAGCUACAGUCGGGCCAGACCGGU & 24 \\
\hline hvu-novel-88 & AGGGACGAUCCGGUUACUAAAC & 22 \\
\hline hvu-novel-89 & AGCGAAGCCGACGGAUGCAUGACC & 24 \\
\hline hvu-novel-9 & UUGAACAUCCCAGAGCCACCG & 21 \\
\hline hvu-novel-91 & AAUUAGACGGUCUAGAUUGCGAGU & 24 \\
\hline hvu-novel-94 & CCGUCGUCGACAUGGCUG & 18 \\
\hline hvu-novel-98 & AAAUGUGAUCUGUACAAAAAAGAC & 24 \\
\hline
\end{tabular}

miRNA $=$ microRNA. 
Table 4. Primer sequences used in the present study

Page 26/36 


\begin{tabular}{|c|c|c|}
\hline MicroRNAs/Genes & Primer name & Primer sequences \\
\hline \multirow[t]{2}{*}{ hvu-miR168-3p } & Forward-Primer & 5'-GATCCCGCCTTGCAC-3 \\
\hline & Universal Reverse & 5'-GTGCAGGGTCCGAGGT-3' \\
\hline \multirow[t]{2}{*}{ hvu-miR156a } & Forward-Primer & 5'-GCAGTGACAGAAGAGAGTGA-3' \\
\hline & Universal Reverse & 5'-GTGCAGGGTCCGAGGT-3' \\
\hline \multirow[t]{2}{*}{ hvu-miR171-5p } & Forward-Primer & 5'-GTGTTGGCTCGACTCAC-3' \\
\hline & Universal Reverse & 5'-GTGCAGGGTCCGAGGT-3' \\
\hline \multirow[t]{2}{*}{ hvu-miR159b } & Forward-Primer & 5'-TTTGGATTGAAGGGA-3' \\
\hline & Universal Reverse & 5'-GTGCAGGGTCCGAGGT-3' \\
\hline \multirow[t]{2}{*}{ hvu-novel-91 } & Forward-Primer & 5'-AATTAGACGGTCTAGATT-3' \\
\hline & Universal Reverse & 5'-GTGCAGGGTCCGAGGT-3' \\
\hline \multirow[t]{2}{*}{ hvu-novel-46 } & Forward-Primer & 5'-AAACTGTGGCTGTCAAAT-3' \\
\hline & Universal Reverse & 5'-GTGCAGGGTCCGAGGT-3' \\
\hline \multirow[t]{2}{*}{ hvu-novel-52 } & Forward-Primer & 5'-GCAGTTCCACAGCTTTCTTG-3' \\
\hline & Universal Reverse & 5'-GTGCAGGGTCCGAGGT-3' \\
\hline \multirow[t]{2}{*}{ Hvu-novel-11 } & Forward-Primer & 5'-TCAGTGCGATCCCTC-3' \\
\hline & Universal Reverse & 5'-GTGCAGGGTCCGAGGT-3' \\
\hline \multirow[t]{2}{*}{$5 S r R N A$ (internal) } & Forward-Primer & 5'-GTCTGCCTGGGTGTCACGCAA-3' \\
\hline & Universal Reverse & 5'-GTGCAGGGTCCGAGGT-3' \\
\hline \multirow[t]{2}{*}{ HORVU4Hr1G027270 } & Forward-Primer & 5'-TCTACCTGCCGTTCTAAGT-3' \\
\hline & Reverse-Primer & 5'-CCGTCCATACCAACATCAA-3' \\
\hline \multirow[t]{2}{*}{ HORVU2Hr1G104640 } & Forward-Primer & 5'-CTCTGCGTGGAAGATTCA-3' \\
\hline & Reverse-Primer & 5'-TGATGAGCTGGACTGACT-3' \\
\hline \multirow[t]{2}{*}{ HORVU3Hr1G067760 } & Forward-Primer & 5 '-GGTCTACATACGGAGCAA-3’ \\
\hline & Reverse-Primer & 5'ACGGATGGAGTAATAAGCA-3' \\
\hline \multirow[t]{2}{*}{ HORVU4Hr1G088470 } & Forward-Primer & 5'-GCTACTAATGTTGGCTCTATC-3' \\
\hline & Reverse-Primer & 5'-TGAATCCTGCTACCTCTTG-3' \\
\hline \multirow[t]{2}{*}{ HORVU7Hr1G083270 } & Forward-Primer & 5'-TACACAACTCCAAGCACTC-3' \\
\hline & Reverse-Primer & 5'-ТACTCAGCACСТССТССТ-3' \\
\hline
\end{tabular}

Page 27/36 


\begin{tabular}{|c|c|c|}
\hline \multirow[t]{2}{*}{ HORVU4Hr1G011590 } & Forward-Primer & 5'-CGATGCCTTAATTGCTACG-3' \\
\hline & Reverse-Primer & 5'-TGTGATTGCCATTGCTGTA-3' \\
\hline \multirow[t]{2}{*}{ HORVU1Hr1G090190 } & Forward-Primer & 5'-TCATCAGCATCACTACACA-3' \\
\hline & Reverse-Primer & 5'-GACACATCACCATCCTCTT-3' \\
\hline \multirow[t]{2}{*}{ HORVU5Hr1G096940 } & Forward-Primer & 5'-GAAGGTGGTGGACGAGAT-3' \\
\hline & Reverse-Primer & 5'-TGAGACGATGGAGGAAGG-3' \\
\hline \multirow[t]{2}{*}{ HORVU5Hr1G096950 } & Forward-Primer & 5'-CGAAGCCGACAAGAAGAG-3' \\
\hline & Reverse-Primer & 5'-TTCAGAAGCAGCGACATAG-3' \\
\hline \multirow[t]{2}{*}{ HORVU7Hr1G073050 } & Forward-Primer & 5'-TTACGGAATGGCTGTCTG-3' \\
\hline & Reverse-Primer & 5'-TTACTTGCGGATACCTCAC-3' \\
\hline \multirow[t]{2}{*}{ TC139057 (internal) } & Forward-Primer & 5'-GAAGGATGAGCAAAAGGCCCT-3' \\
\hline & Reverse-Primer & 5'-GGCAGGCAGACTCATTTCTTCC-3' \\
\hline
\end{tabular}

\section{Figures}


a

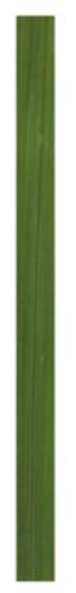

KL14N

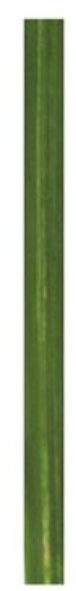

KL14S

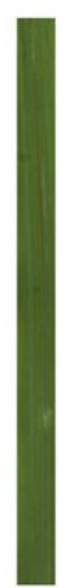

ZN

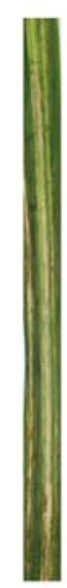

ZS

C

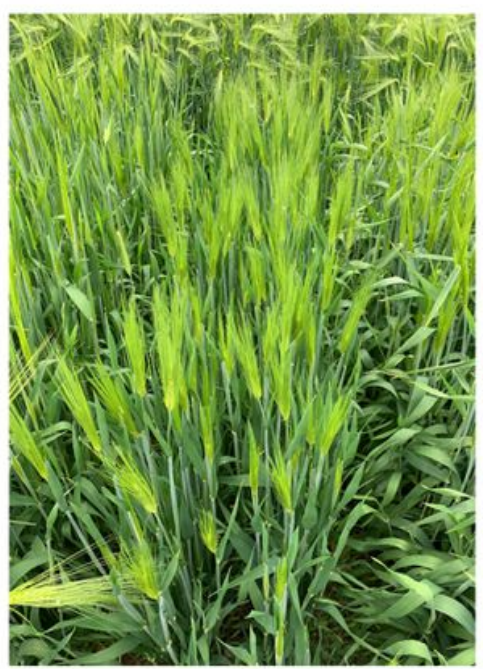

Kunlun14

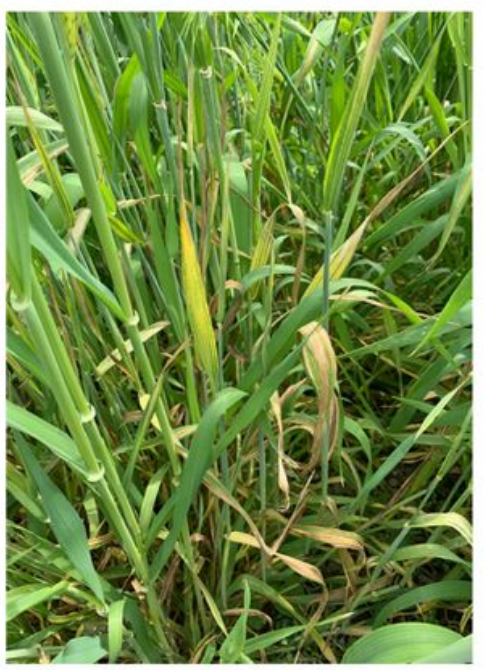

Z1141

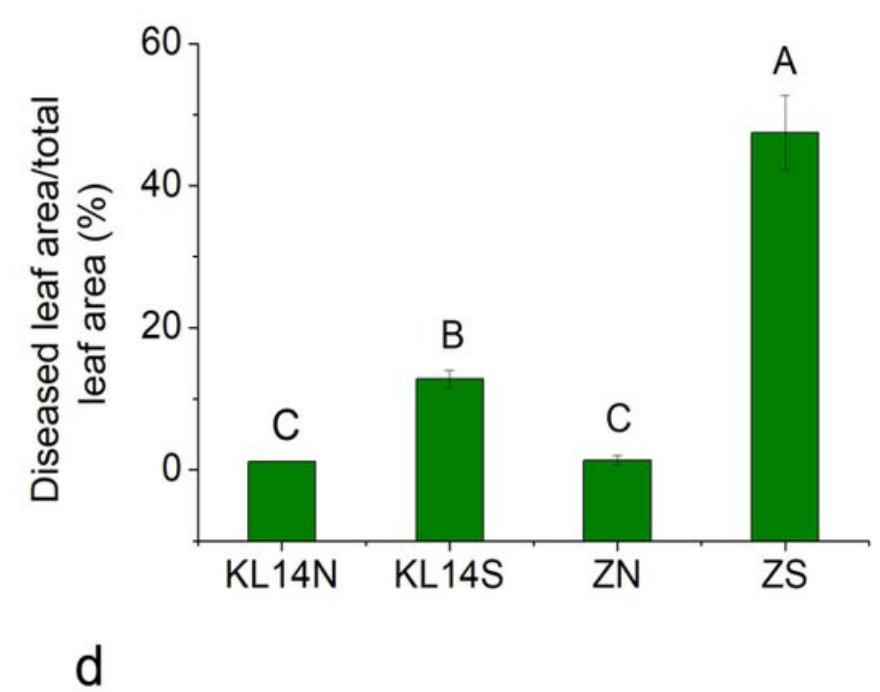

b

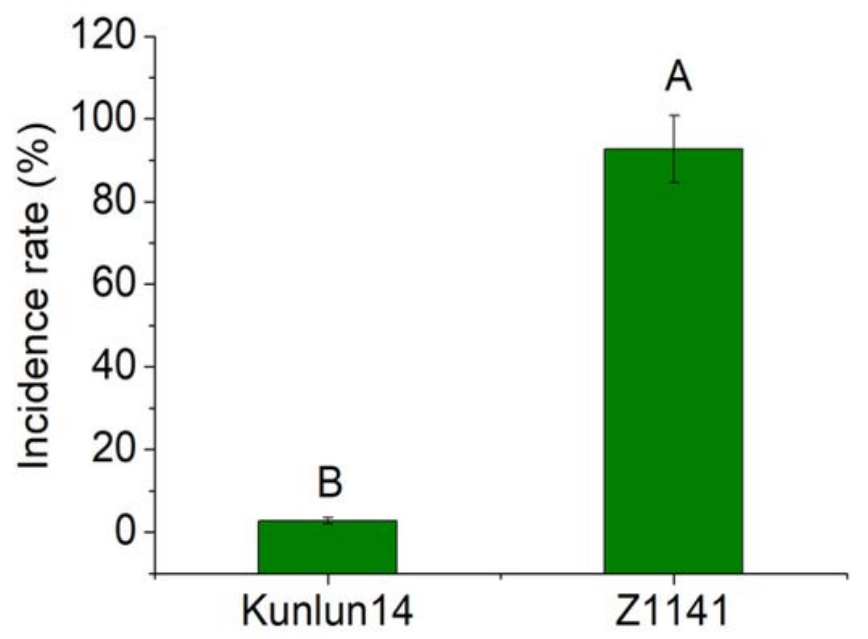

\section{Figure 1}

Visible differences in leaves infected by BLS in two genotypes of Tibetan hulless barley plants. a, Phenotype of KL14N, KL14NS, ZN, and ZS. b, The ratio of diseased leaf area to total leaf area of KL14N, KL14NS, ZN, and ZS. c, Phenotype of the epidemic area of Kunlun14 and Z1141. d, the epidemic area of Kunlun14 and Z1141. Bars in B and D are means \pm SD of three biological replicates; means labeled with different letters are significantly different at $P<0.01$ (Tukey's test). BLS = barley leaf stripe; KL14N = 'Kunlun14' without barley leaf stripe; KL14S = 'Kunlun14' with barley leaf stripe; SD = standard deviation; ZN = 'Z1141' without barley leaf stripe; ZS = 'Z1141' with barley leaf stripe. 


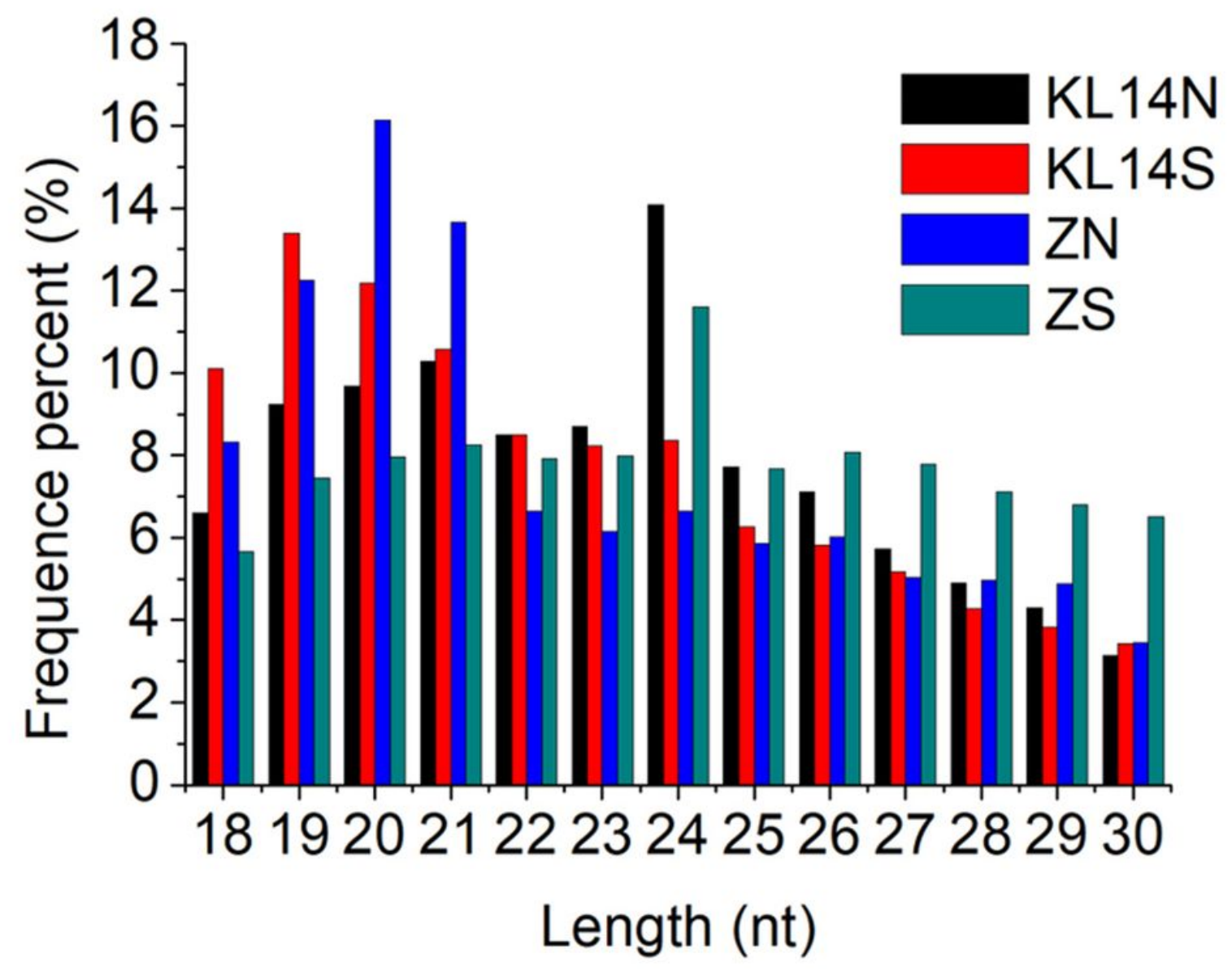

Figure 2

Length distribution of small RNAs in the control and BLS-infected leaves of Z1141 and Kunlun14. BLS = barley leaf stripe; KL14N = 'Kunlun14' without barley leaf stripe; KL14S = 'Kunlun14' with barley leaf stripe; ZN = 'Z1141' without barley leaf stripe; ZS = 'Z1141' with barley leaf stripe.

a
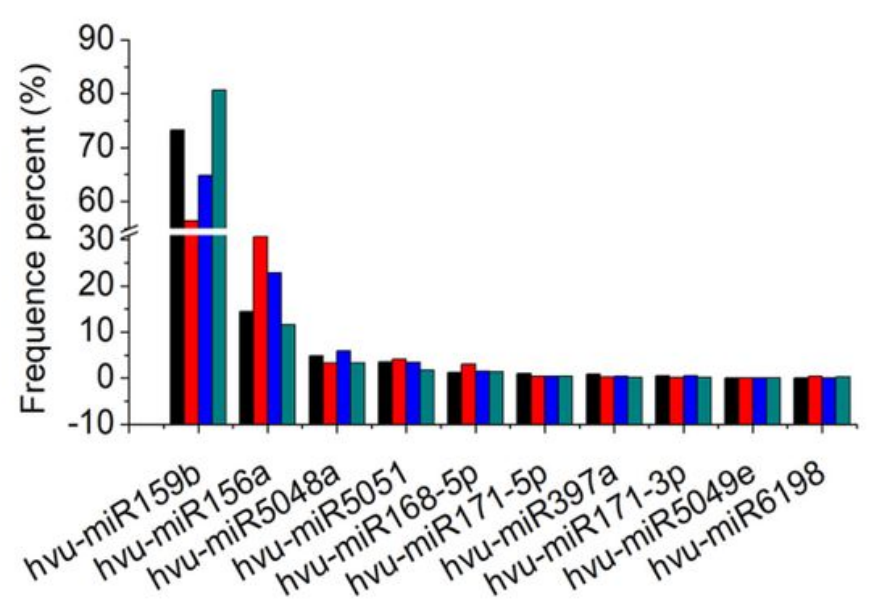

b

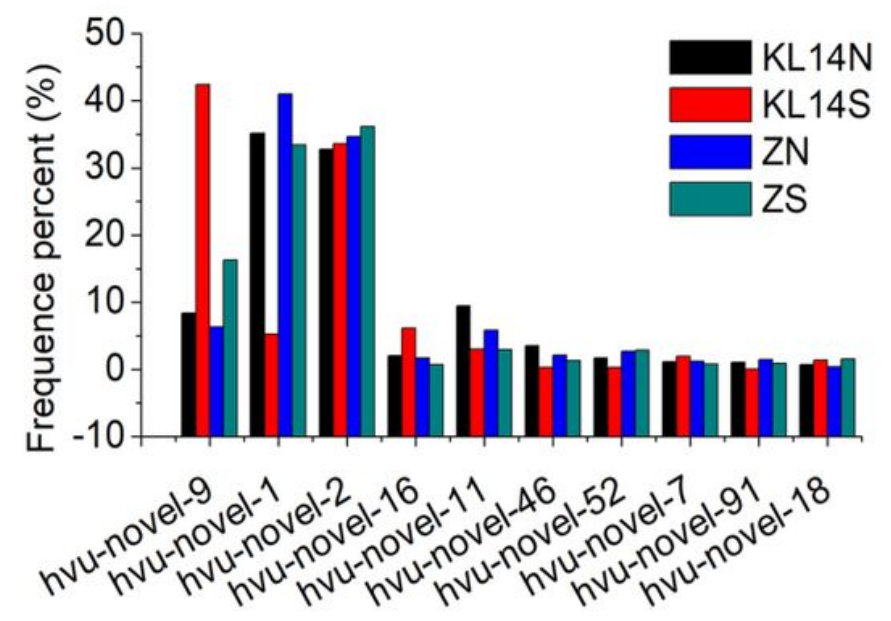




\section{Figure 3}

Abundance of the 10 most highly expressed conserved and novel miRNAs in the KL14N, KL14NS, ZN, and ZS Tibetan hulless barley libraries. a, Conserved miRNAs. b, Novel miRNAs. KL14N = 'Kunlun14' without barley leaf stripe; KL14S = 'Kunlun14' with barley leaf stripe; miRNA = microRNA; ZN = 'Z1141' without barley leaf stripe; ZS = 'Z1141' with barley leaf stripe.

a

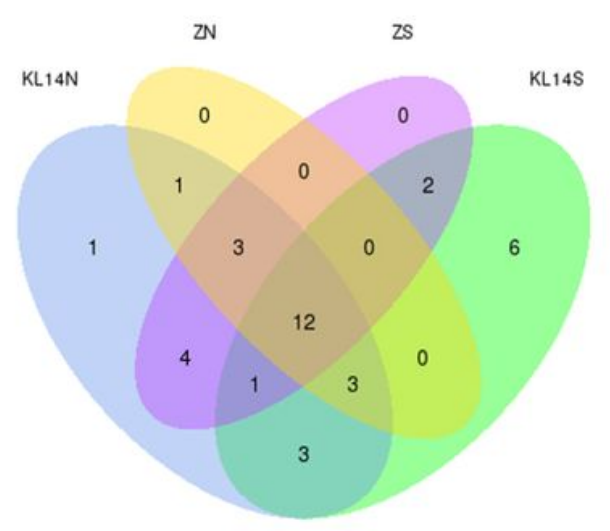

b

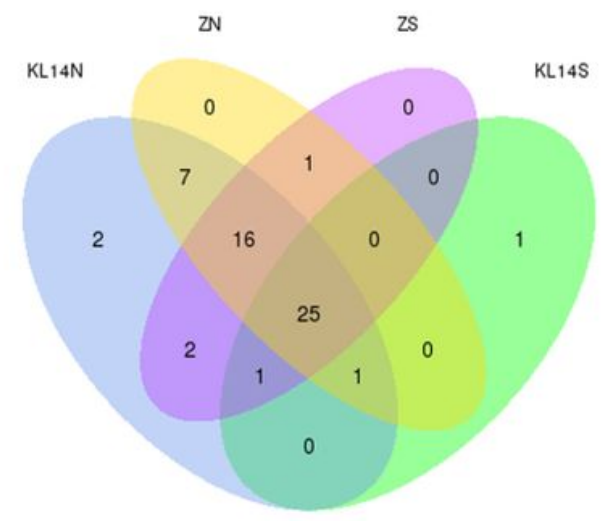

C

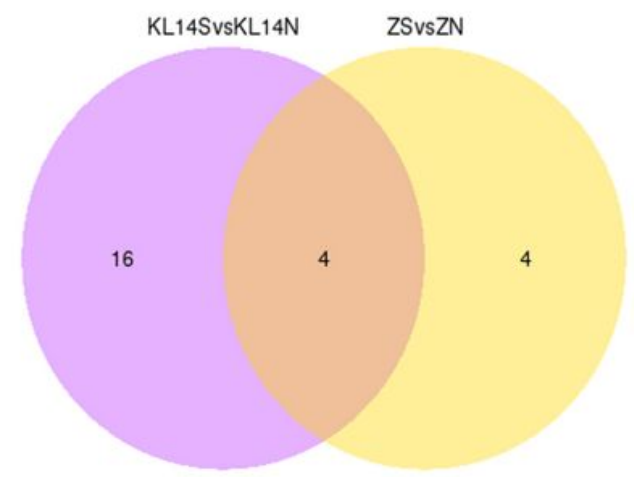

\section{Figure 4}

Venn diagrams of Tibetan hulless barley miRNAs identified in the four libraries. A, Conserved miRNAs. B, Novel miRNAs. C, Venn diagrams of miRNAs before and after BLS infection of the Kunlun14 and Z1141 plants' leaves. BLS = barley leaf stripe; KL14N = 'Kunlun14' without barley leaf stripe; KL14S = 'Kunlun14' with barley leaf stripe; miRNA = MicroRNA; ZN = 'Z1141' without barley leaf stripe; ZS = 'Z1141' with barley leaf stripe. 


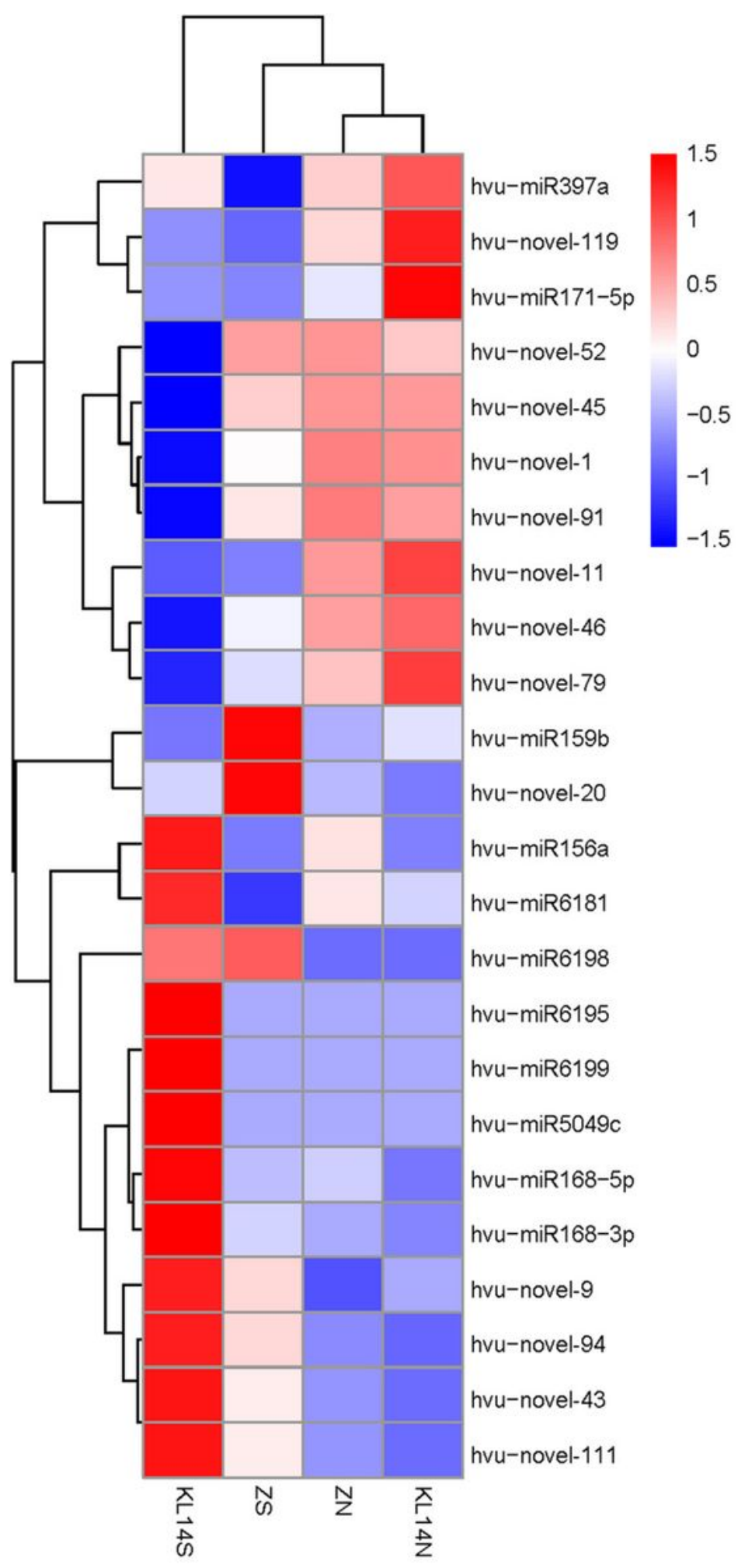

Figure 5

Heatmap showing the differentially expressed miRNAs of KL14N, KL14S, ZN, and ZS. The color scale indicates the log2-transformed values of the measured fold changes. KL14N = 'Kunlun14' without barley leaf stripe; KL14S = 'Kunlun14' with barley leaf stripe; miRNA = microRNA; ZN = 'Z1141' without barley leaf stripe; ZS = 'Z1141' with barley leaf stripe. 


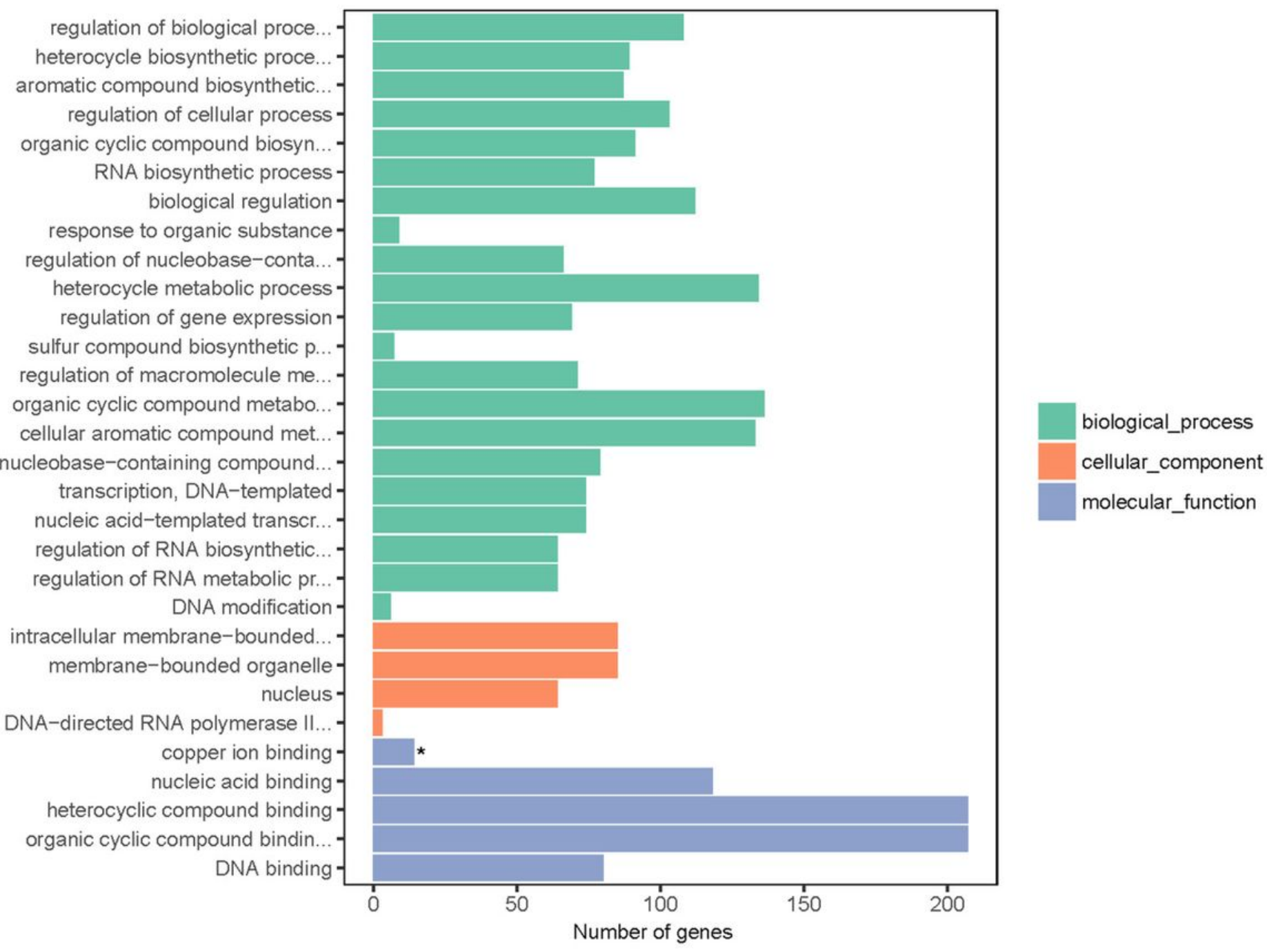

Figure 6

GO enrichment analysis of the target genes of four miRNAs responsive to BLS in Tibetan hulless barley. $\mathrm{BLS}$ = barley leaf stripe; $\mathrm{GO}=$ gene ontology; $\mathrm{miRNA}=$ microRNA. 


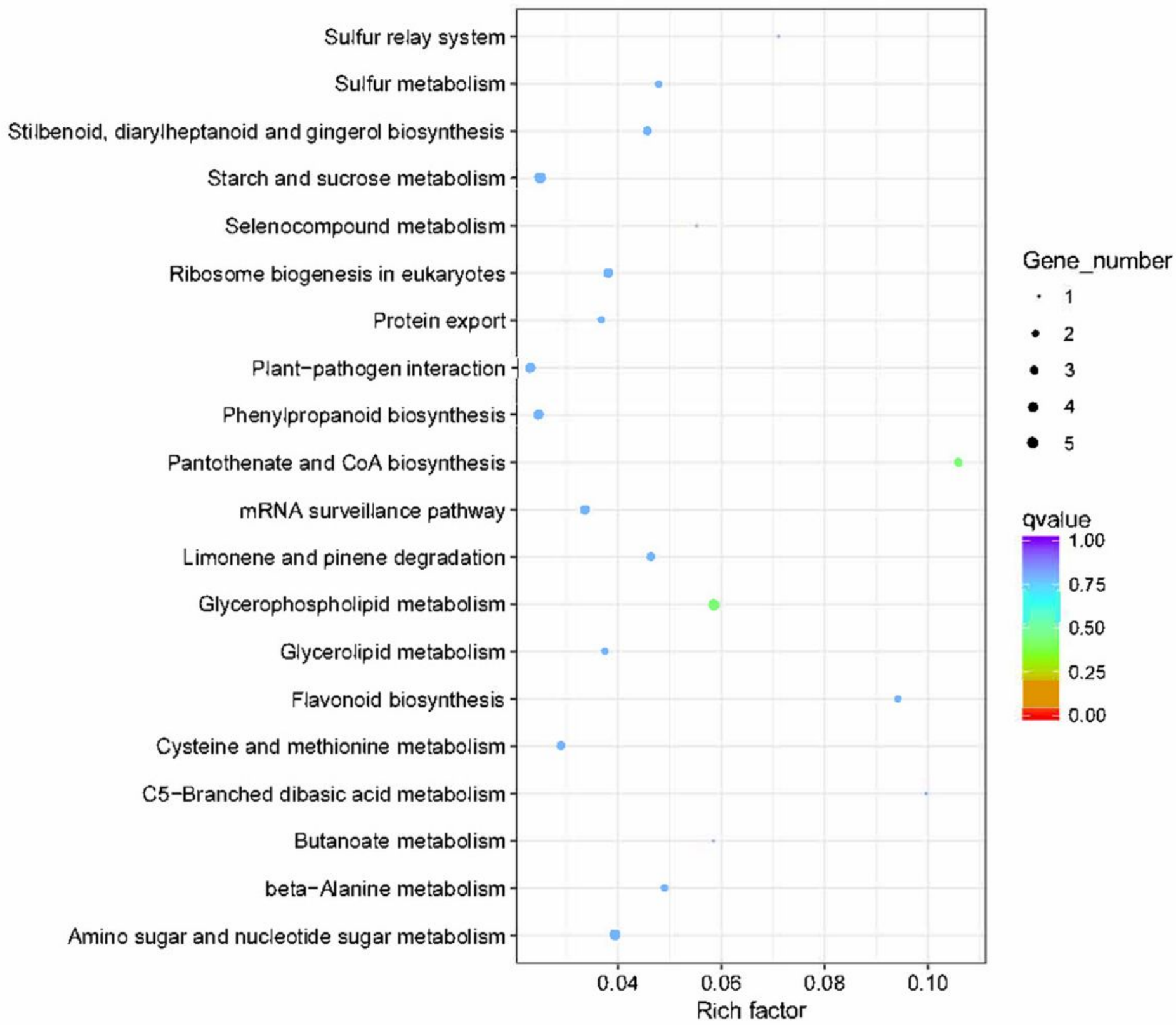

\section{Figure 7}

KEGG analysis of target genes of four miRNAs responsive to BLS in Tibetan hulless barley. BLS = barley leaf stripe; KEGG = Kyoto Encyclopedia of Genes and Genomes; miRNA = microRNA. 


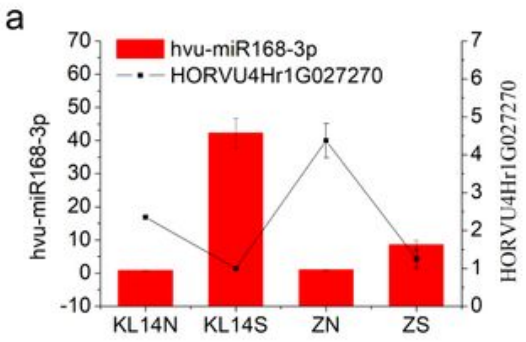

C

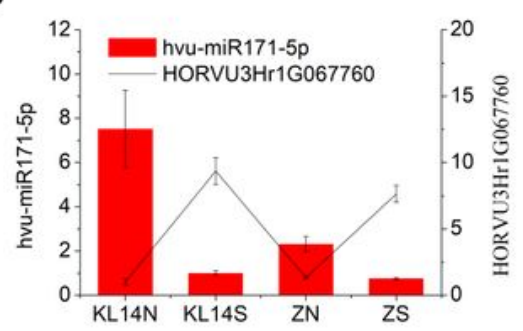

e

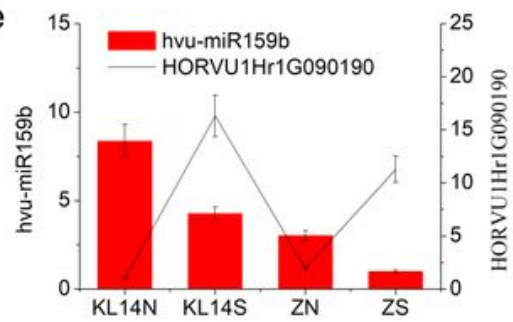

g
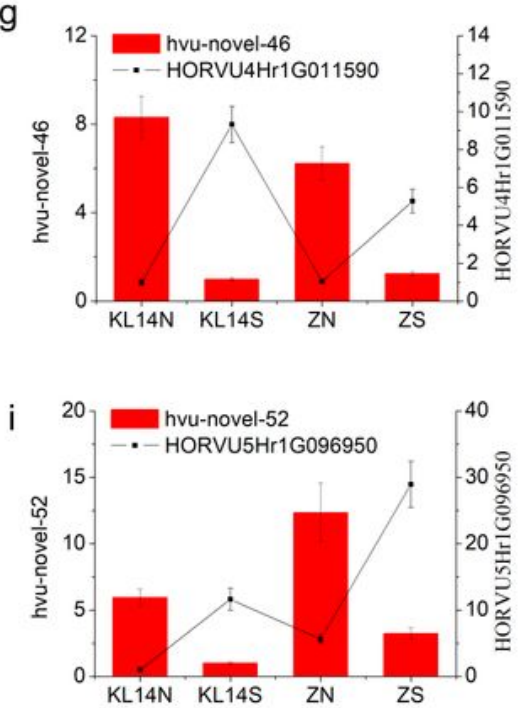

b

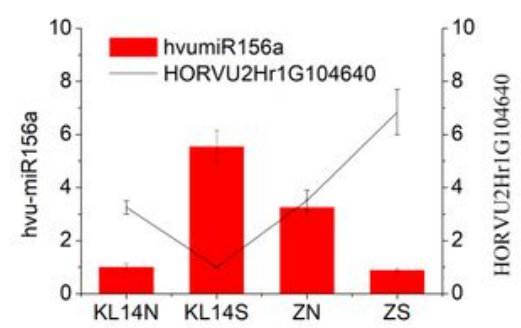

d

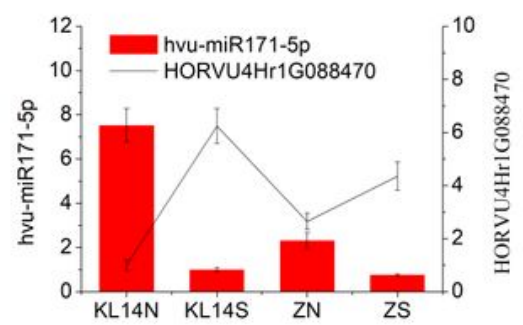

f

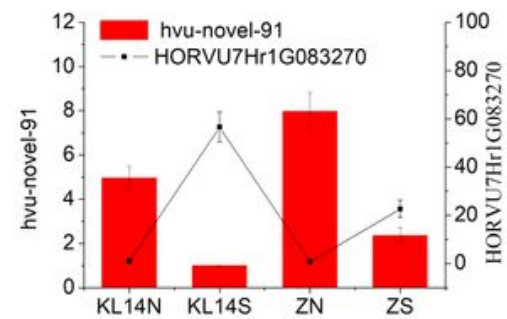

h
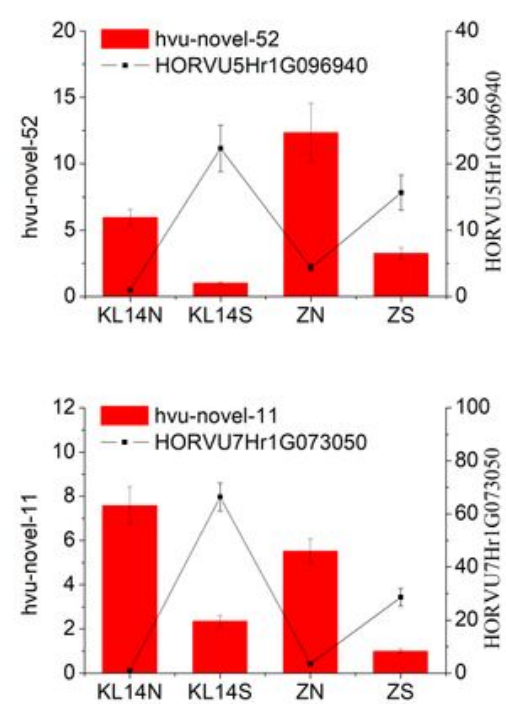

\section{Figure 8}

Comparison of expression levels of eight miRNAs and ten target genes in leaves of KL14N, KL14S, ZN and ZS by qRT-PCR. The red histogram represented the relative expression values of miRNA by qRT-PCR, the black line represented the relative expression values of target genes by qRT-PCR. a-j, qRT-PCR analysis of miRNAs and their target genes responsed to BLS. The 5SrRNA and TC139057 were used as internal gene of miRNAs and target genes. The data displayed in the histograms are expressed as the 
means \pm SD. $B L S=$ barley leaf stripe; KL14N = 'Kunlun14' without barley leaf stripe; KL14S = 'Kunlun14' with barley leaf stripe; $m i R N A=$ microRNA; qRT-PCR = quantitative real-time PCR; SD = standard deviation; ZN = 'Z1141' without barley leaf stripe; ZS = 'Z1141' with barley leaf stripe.

\section{Supplementary Files}

This is a list of supplementary files associated with this preprint. Click to download.

- Additionalfile1.docx

- Additionalfile2.docx

- Additionalfile3.docx

- Additionalfile4.docx

- Additionalfile5SupplementaryFigure1.jpg

- Additionalfile6.xlsx

- Additionalfile7.xlsx

- Additionalfile8SupplementaryFigure2.jpg

- Additionalfile9.xlsx

- Additionalfile10.xlsx

- Additionalfile11.doc 